San Jose State University

SJSU ScholarWorks

Master's Projects

Master's Theses and Graduate Research

Winter 2021

\title{
Differential Gene Expression Analysis of Rodents Exposed to Long-Term Space Flight and Insights into Physiological Effects
}

Jonathan Oribello

San Jose State University

Follow this and additional works at: https://scholarworks.sjsu.edu/etd_projects

Part of the Bioinformatics Commons

\section{Recommended Citation}

Oribello, Jonathan, "Differential Gene Expression Analysis of Rodents Exposed to Long-Term Space Flight and Insights into Physiological Effects" (2021). Master's Projects. 976.

DOI: https://doi.org/10.31979/etd.d4e7-x2g3

https://scholarworks.sjsu.edu/etd_projects/976

This Master's Project is brought to you for free and open access by the Master's Theses and Graduate Research at SJSU ScholarWorks. It has been accepted for inclusion in Master's Projects by an authorized administrator of SJSU ScholarWorks. For more information, please contact scholarworks@sjsu.edu. 
Differential Gene Expression Analysis of Rodents Exposed to Long-Term Space Flight and Insights into Physiological Effects

\author{
A Project \\ Presented to \\ Department of Computer Science \\ San José State University \\ In Partial Fulfillment \\ Of the Requirements for the Degree
}

By

Jonathan Oribello

November, 2020 


\begin{abstract}
Space travel presents inherent risks to human health and a better understanding of space biology is required to mitigate harm in an oncoming age of increased space travel. Omics analysis will play a central role in better understanding human health in space. In this project, differential gene expression analysis was performed on GLDS-104, an open science dataset provided by NASA's GeneLab.

GeneLab's RNA-Seq Consensus Pipeline was implemented using Nextflow, performed on San Jose State University's College of Science High Performance Computing Cluster, and optimized for computational resource efficiency. Comparison of the Nextflow implemention developed in this project to GeneLab's posted results for the pipeline indicate a high degree of reproducibility was achieved. The results from GLDS-104 were further analyzed to develop insights into the physiological effect of space flight on skeletal muscle and demonstrate utility of the pipeline. Among the insights discussed includes a theory suggesting the upregulation in myogenesis related genes during space flight compared to hindlimb unloading may be due to unique space flight ambulation. This project highlights the importance of connecting effective pipeline specifications, efficient workflow implementations, and high performance computing to enable space biology omics research.
\end{abstract}

Keywords: microgravity, muscle atrophy, Nextflow 


\section{ACKNOWLEDGEMENTS}

I would like to acknowledge and thank the following individuals for their role in the completion of this project.

- Dr. Phillip Heller for guidance, support and introducing me to the exciting field of space biology.

- Dr. Wendy Lee and Dr. Martina Bremer for their feedback and their teachings that enabled me to tackle this project.

- Dr. Amanda Saravia-Butler from GeneLab for guidance and insights while developing the Nextflow implementation of the GeneLab RNA-Seq Consensus Pipeline.

- Steve Boring for advice on best practices when using the College of Science HPC.

- My M.S. Bioinformatics peers for enriching discussions and support in navigating our program.

- My friends and family for their understanding, encouragement and support during the program. 


\section{Table of Contents}

I. Introduction .6

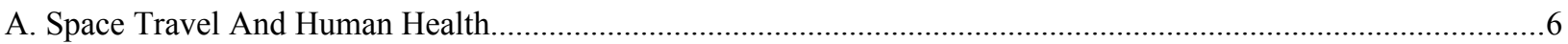

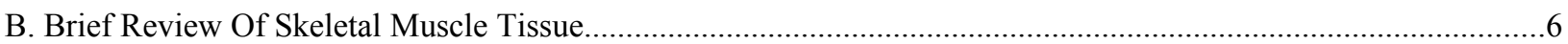

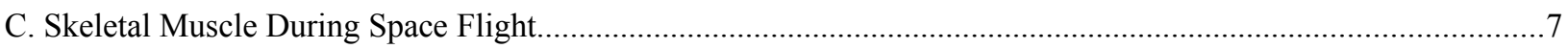

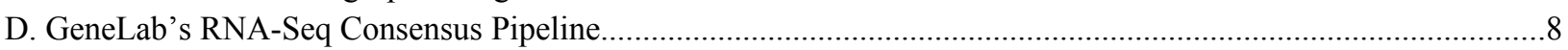

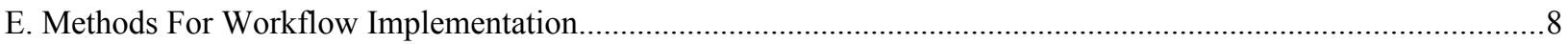

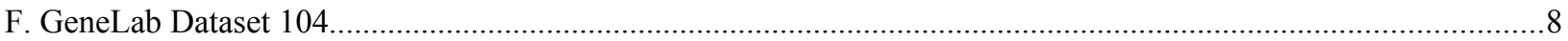

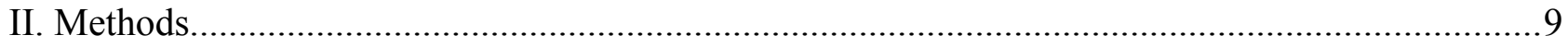

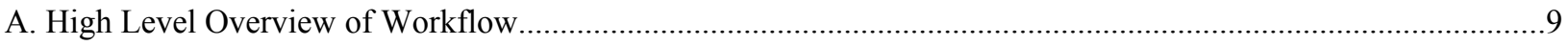

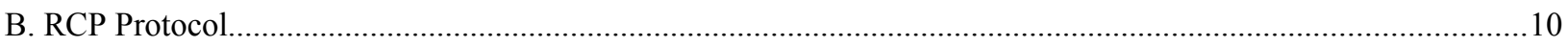

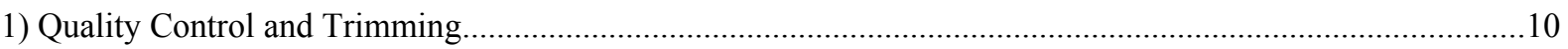

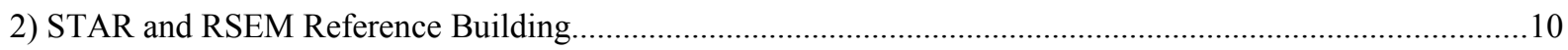

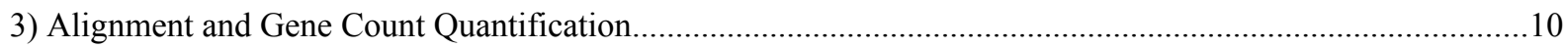

4) Normalization and Differential Gene Expression Analysis Using Adapted DESeq2 Script...........................10

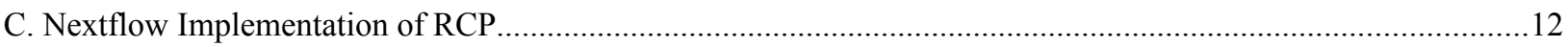

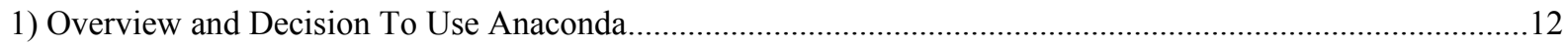

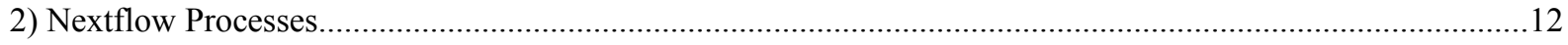

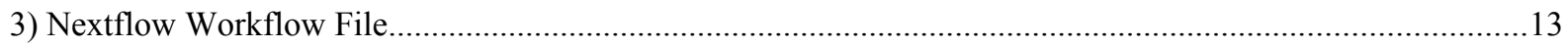

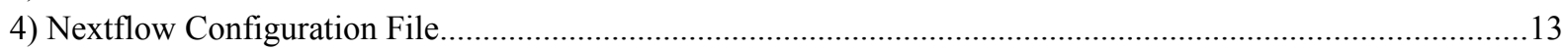

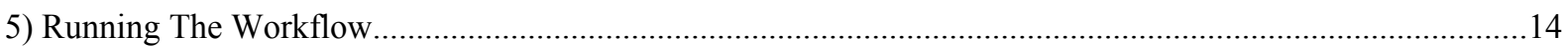

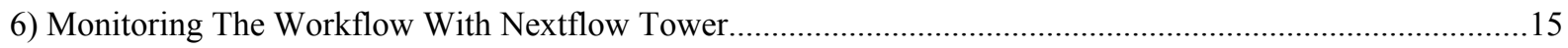

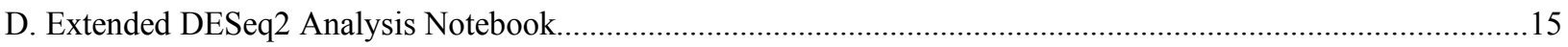

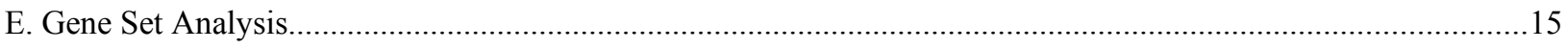

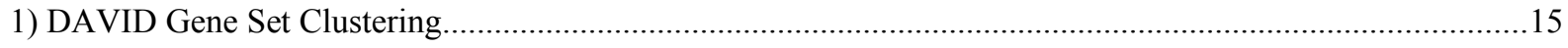

2) Preranked Gene Set Enrichment Analysis.........................................................................................

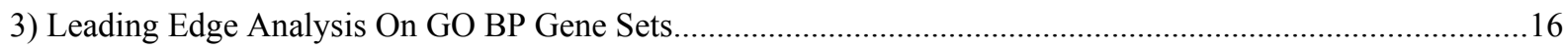

4) Cytoscape Enrichment Map Of GO Biological Processes...........................................................................16



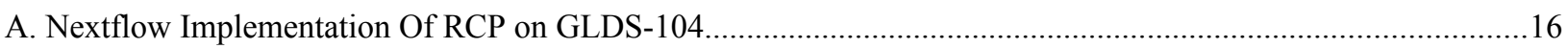

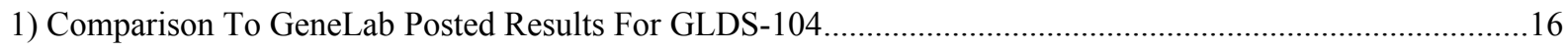

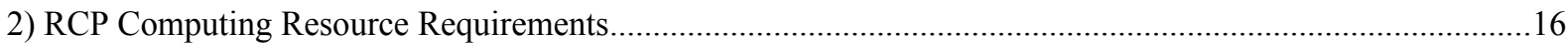

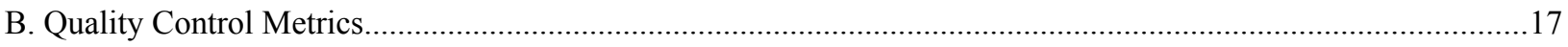

C. Difference In Requested Read Lengths And Observed Read Lengths...........................................................17

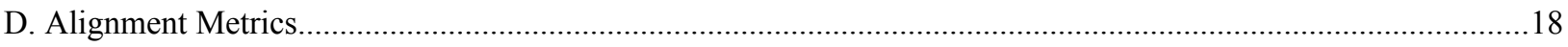

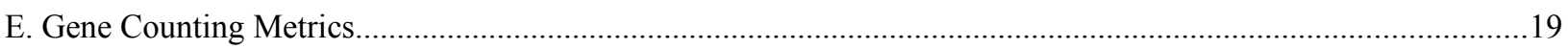

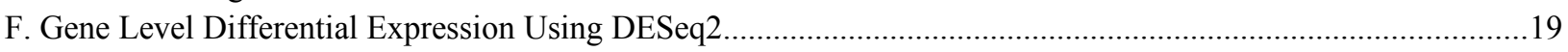

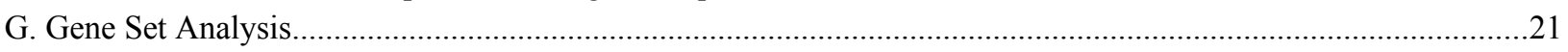

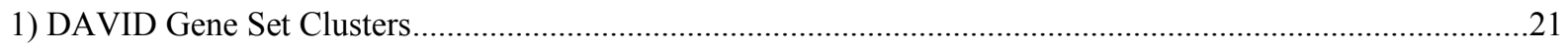

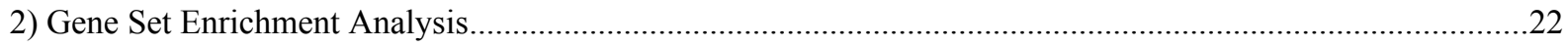

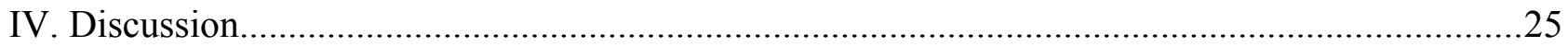

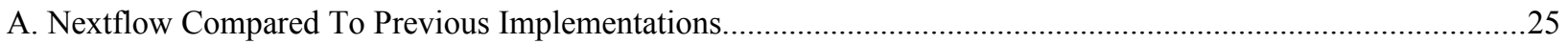

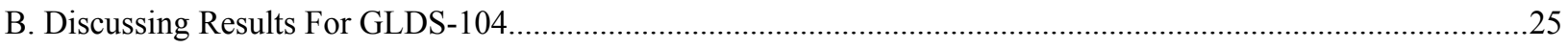

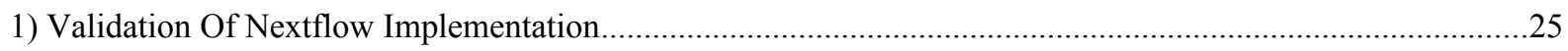

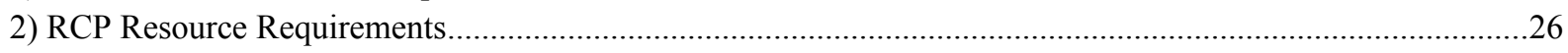

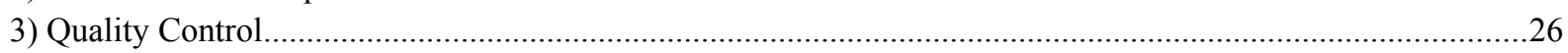

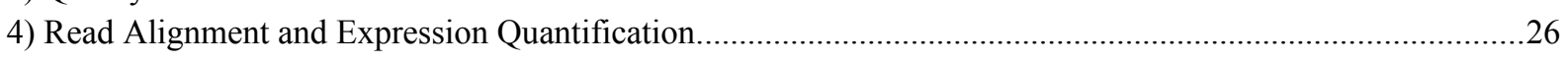




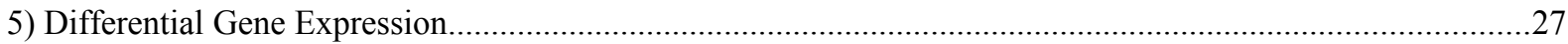

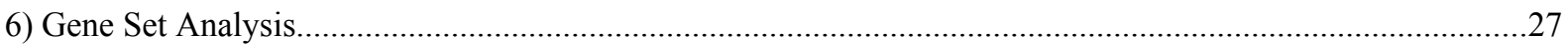

a) DAVID enriched gene set clusters and relation to muscle tissues.....................................................27

b) Enrichment of upregulated genes in hallmark myogenesis gene set....................................................27

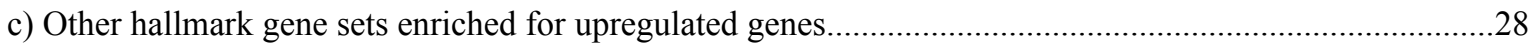

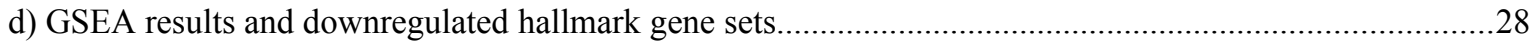

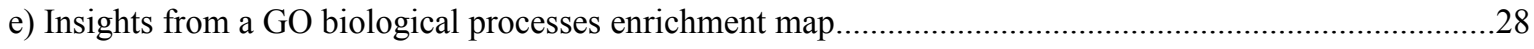

f) Heat shock protein expression downregulation and connection to muscle atrophy.................................29

g) Possible influence of circadian rhythm disruption and separation from normal geomagnetic field on skele-

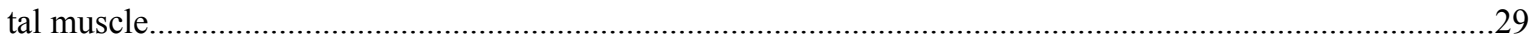

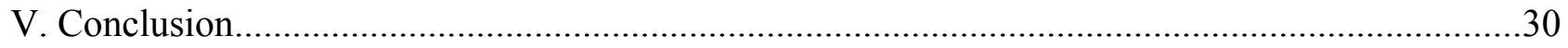

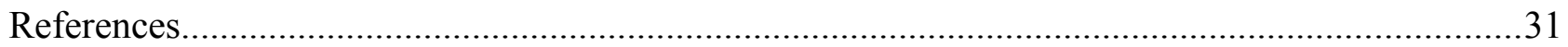




\section{Table of Figures}

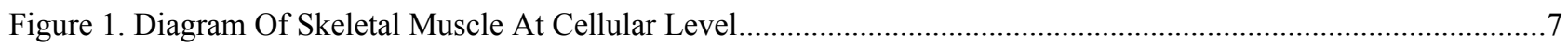

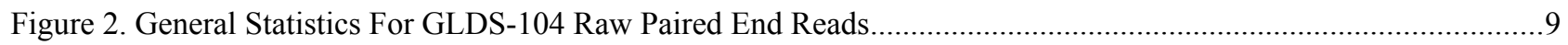

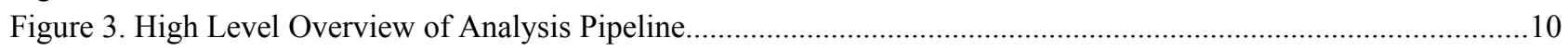

Figure 4. DESeq2 Dispersion Estimation Process For Subsampled Example Dataset.................................................11

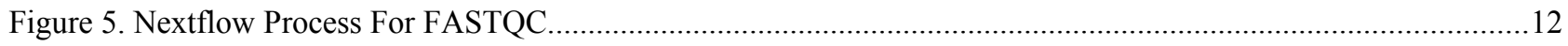

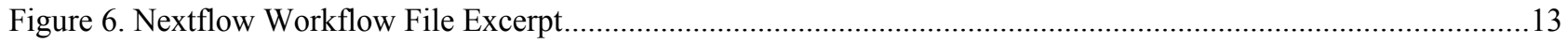

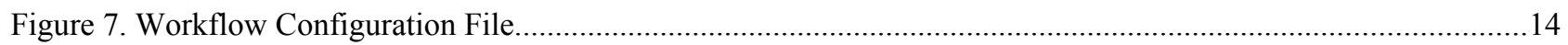

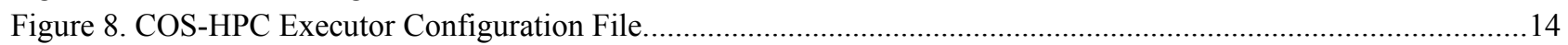

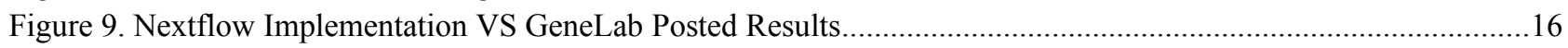

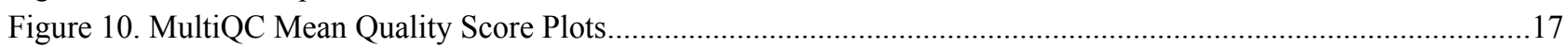



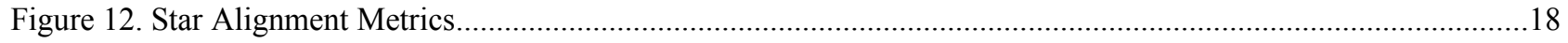

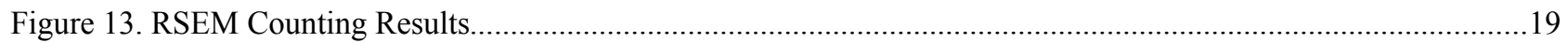

Figure 14. Sankey Diagram For Genes Processed Through DESeq2 ..................................................................19

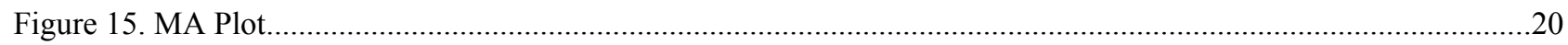

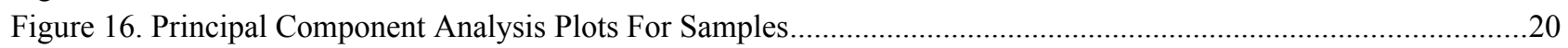

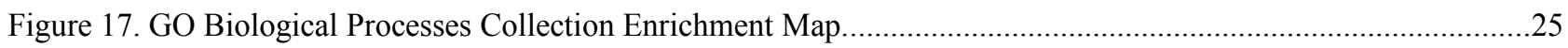




\section{Index of Tables}

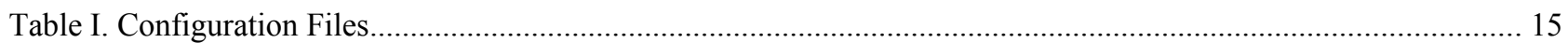

Table II. 4 Genes Uniquely Detected As Differentially Expressed In Nextflow............................................................17

Table III. 17 Genes Uniquely Detected As Differentially Expressed In GeneLab Posted Results..................................17

Table IV. Summary of Resources For RCP Performed on COS-HPC For GLDS-104 .............................................18

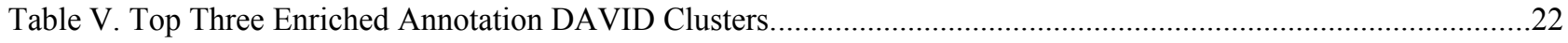

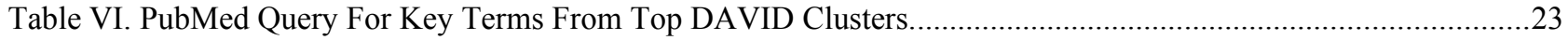



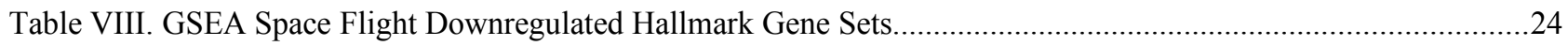

Table IX. Prevalent Genes From Leading Edge Analysis On 611 GO BP Gene Sets......................................................25 


\section{INTRODUCTION}

\section{A. Space Travel And Human Health}

Space travel is one of humanity's most ambitious goals. Driven by exciting technological and scientific advances, both government space agencies and the private space industry are making strides towards a return to the moon by 2024 and the first manned missions to Mars by 2034 [1]. Additionally, civilian opportunities to go to space are anticipated to quickly increase in tandem with growth in the private space industry [2] . Recent advances promise two things about space travel. First, reaching further into space will require longer space travel times. Current estimates place the travel time for round trips to Mars in excess of an entire year [3]. Second, increasing access to space for Earth orbit missions exposes both professionals and civilians to large amounts of space travel over a series of trips. Ultimately, this suggests we are entering an age where more people will have access to space and for increasingly longer amounts of time compared to the entire human history of space travel. While this promises a boon of advancements for mankind, this also raises significant questions about the human body in space. What are the harmful human health effects inherent to space travel? How can we mitigate or prevent the impact of these space travel health problems?

Space travel health problems have been studied since the first manned flights in the early 60's [4]. At the time, biological measurements of the astronauts were collected to assess the impact of space travel on the body. In recent years, the health impacts observed in those earliest trips were found to be caused mainly by non-space specific factors (e.g. time spent in heavy spacesuits); however these first trips exposed astronauts to less than two days of travel [4]. Modern missions with lengthier space travel have been studied extensively and with increasingly sophisticated methods. Recent studies have connected multi-week and multi-month space travel missions to detrimental affects on the body including muscle and bone mass loss, immune system suppression, and even disruptions to neurological system communication [5]-[8]. Space travel inherently carries a set of well documented and unknown environmental factors that affect human health. Among the most studied factors are exposure to microgravity and increased exposure to radiation. One solution would be to limit exposure to microgravity and radiation during space travel. While this may serve to alleviate health issues, full or even significant reduction in exposure is likely impractical. A second solution is developing biological countermeasures to address the harmful effects caused by space travel factors. In either case, development of countermeasures hinges on a comprehensive understanding of human health in space.

Towards this goal, NASA has launched a series of Rodent Research Missions that explore the effect of space flight on human health by first examining the effects on model organisms [9]. These missions have explored a number of analytical routes including metablomics and proteomics for a variety of tissues extracted from space flight rodents. This project will focus on transcriptomics using RNA-Seq with the following goals: First, implement a well validated RNA-Seq based differential gene expression pipeline and assess accuracy of the implementation. Second, use the results of the implemented pipeline to develop biologically significant insights about the effect of space flight on skeletal muscle tissue.

\section{B. Brief Review Of Skeletal Muscle Tissue}

Skeletal muscle tissue is central to a number of crucial biological functions. The ability to walk, run, manipulate and even stand upright are all made possible by skeletal muscle. Skeletal muscle, depicted in Fig. 1, is organized as bundles of myocytes which in turn are enclose bundles of myofibrils. These myofibrils generate force through a contractile molecular mechanism. This contractile mechanism is kick started by a 
release of calcium ion from the sarcoplasmic reticulum organelle. The increase in calcium ion concentration within the myocyte removes inhibitory molecules surrounding the myofibrils. Finally, a sliding motion powered by ATP is achieved between myosin and actin filaments causing a contraction of myofibrils [10]. The magnitude and sustainability of force generation is controlled by a complex biological network involving mitochondrial abundance, metabolism, nutrition, muscle fiber composition and muscle use patterns.

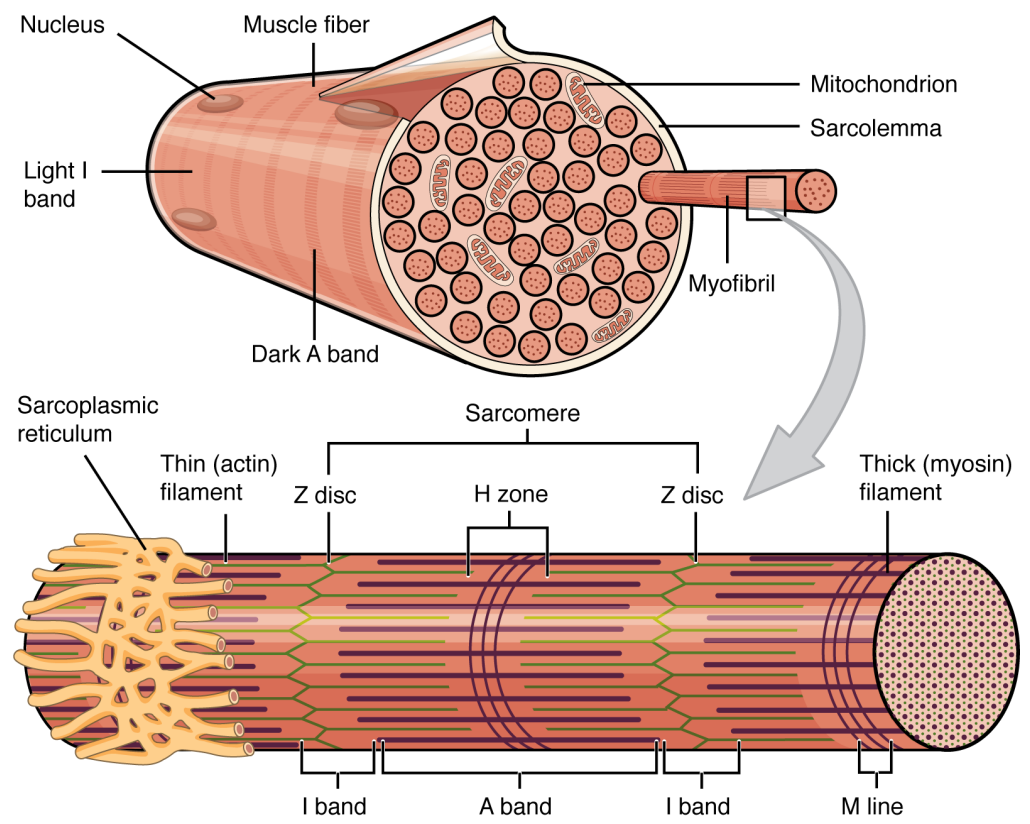

Figure 1. Diagram Of Skeletal Muscle At Cellular Level Adapted from [11].

\section{Skeletal Muscle During Space Flight}

Skeletal muscle is drastically affected by space flight. Most markedly, astronauts experience a loss of muscle mass as a consequence of space flight [12]-[14]. This muscle atrophy reduces the ability for muscles to generate force and negatively impacts the ability for an astronauts to perform basic and essential tasks. A full understanding of muscle atrophy and space flight still remains lacking; however, perturbations to bioenergetics, muscle fiber composition, and skeletal muscle proteostasis have been observed and play a key role in muscle atrophy [15]-[19].

Bioenergetic balance, largely a function of mitochondria abundance and metabolism, is found to be perturbed during space flight [20]-[23]. This reflects changes to energy requirements caused by the removal of normal gravity loading, but also reflects more complex changes to overall metabolism during space flight including shifts in metabolic processes and changes to energy intake [24].

Muscle fiber composition changes are observed during space flight [19], [25]. These fiber composition changes can be characterized as an overall shift from slow twitch fibers to fast twitch fibers. Slow twitch fibers are associated with sustained, lower magnitude force generation consistent with activites like standing. In contrast, fast twitch fibers are associated with fleeting bursts of higher magnitude force generation [10]. Fiber composition shifts have been associated with adaption to changes in functional demands on muscles including those enountered when going from normal gravity to microgravity environments.

Normal function for skeletal muscle requires maintaining protein levels and structures appropriate for current demands for force generation. This proteostasis is disrupted by space flight including increases in catabolic rates, decreases in anabolic rates, and changes to chaperone protein expression [15], [16].

The reduction of skeletal muscle function presents an enormous challenge for the future of space travel. Correction and/or prevention of space flight induced muscle changes will be key to enabling space travelers to function and stay healthy during and after space flight. A driving factor in addressing this challenge continues to be the development of robust analytical methods and pipelines to enhance our cur- 
rent understanding of space biology.

\section{GeneLab's RNA-Seq Consensus Pipeline}

GeneLab is a NASA project that hosts space biology omics datasets and establishes pipelines and protocols for analyzing such datasets [26]. This includes a pipeline named the RNA-Seq Consensus Pipeline (RCP). The RCP was designed by a broad community of spaceflight researchers to analyze RNASeq data for transcriptome profiling and is documented in a recently released preprint [27]. The article specifies an entire processing pipeline, from raw read files to differential gene expression results. The major steps of the pipeline are quality control reporting, adaptor removal, alignment to transcriptome, quantification of expression and finally differential gene expression analysis. While the article specifies every step and software in the process, there exists a gap between pipeline specification and an executable workflow implementation.

\section{E. Methods For Workflow Implementation}

Implementing the RCP using a workflow tool will allow researchers to efficiently scale to available computing resources and reproduce analysis with ease. Using a standard workflow tool also allows other researchers familiar with the tool to modify or extend the RCP. With these goals in mind, there exist a number tools available for implementing a workflow.

The simplest method reviewed in this project was implementation via Bash scripts. Each step of the RCP is ultimately launched from a command line and these commands can be encoded as Bash scripts. A disadvantage of using Bash scripts is that workflow features including configuration, workflow monitoring, version controlling, software management, and parallel execution must be manually implemented. While this is possible, any manual implementation carries the risk of introducing bugs and requires other users to learn the custom manual implementation. In the context of allowing other researchers to reuse, modify and extend the RCP, this is not a viable option.

Another implementation method explored was using Snakemake. Snakemake is a Python based workflow engine [28]. Unlike Bash scripting, Snakemake includes key workflow features like software management, parallel execution and execution environment configuration. While these features made Snakemake a potential candidate for RCP implementation, a third implementation approach was found to more promising.

The third and final implementation software explored was Nextflow. Nextflow is a Groovy based workflow engine [29]. In addition to the features available in Snakemake, Nextflow also includes integration with GitHub for version control, live workflow execution monitoring with Nextflow Tower and a modular process system. The Nextflow community also appeared more active than the Snakemake community. As a result, Nextflow has more fully established best practices and ongoing support for new and experienced users. Nextflow was ultimately chosen as the approach for this project due to its unique features, extremely helpful community, and growing usage in omics analysis [29]. In addition to a Nextflow implementation of the RCP, a San Jose State University's College of Science High-Performance Computing Cluster (COS-HPC) specific configuration was created to take advantage of high performance computing power. This configuration takes into account the computing arrangement on the COS-HPC to efficiently leverage the cluster's resources.

\section{F. GeneLab Dataset 104}

Upon advice from Dr. Saravia-Butler at GeneLab, GeneLab Dataset 104 (GLDS-104) was chosen as the case study for this project as soleus muscle tissue has been shown in previous studies to be significantly affected by space flight [19], [25]. GLDS-104 contains raw and processed data from the soleus muscle of 12 C57BL/6J mice that were part of NASA's Rodent Research Mission 1.

A summary of the experimental protocol is as follows: Six mice were brought aboard the International Space Station for 37 days before sacrifice in space. The other six mice served as controls and were 
kept on Earth in the same kind of housing. After sacrifice, the soleus muscle was excised from each animal. Next, the tissue was used to prepare an RNA library after reduction of rRNA. Finally, the library was sequenced using the Illumina HiSeq 4000 platform to generate 100 base pair paired end reads [30]. Fig 2. depicts general information related to the paired end reads.
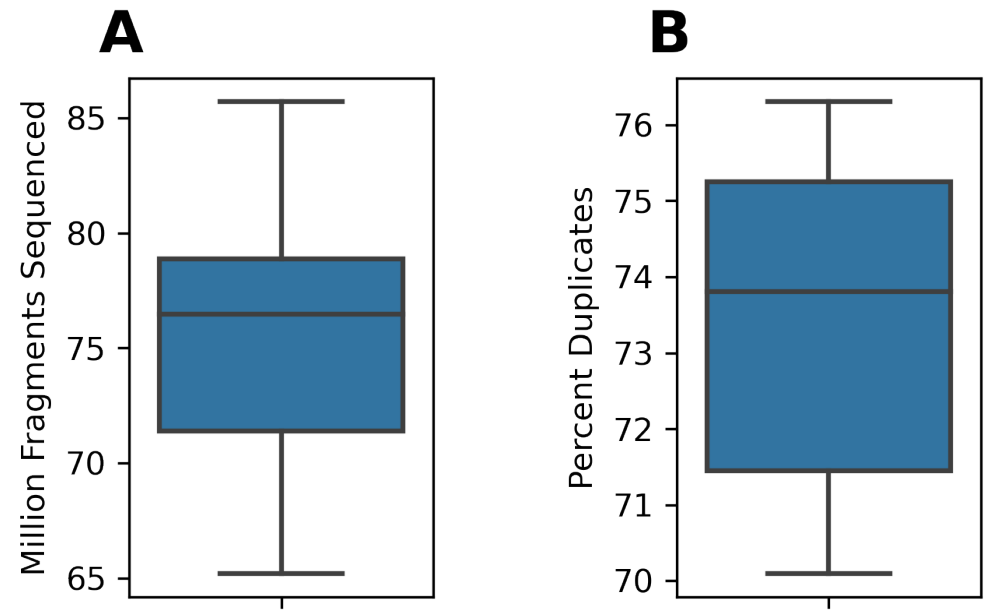

Figure 2. General Statistics For GLDS-104 Raw Paired End Reads (A) Boxplot of Fragments Sequenced Per Sample (B) Boxplot of Percent Duplicates Per Sample Estimated By FastQC

\section{METHODS}

\section{A. High Level Overview of Workflow}

This project implements the RCP to generate gene-level counts as well as standard differential expression analysis using DESeq2 [27], [31] . The pipeline was performed on the COS-HPC. The processed data generated from the RCP are further analyzed using DESeq2 and gene set analysis methods, DAVID and GSEA, to develop insights from the entire collection of significantly differentially expressed genes [31]-[33] . Fig. 3 presents a high level flowchart for the RCP implemented in Nextflow as well as the extended analysis performed on GLDS-104. 


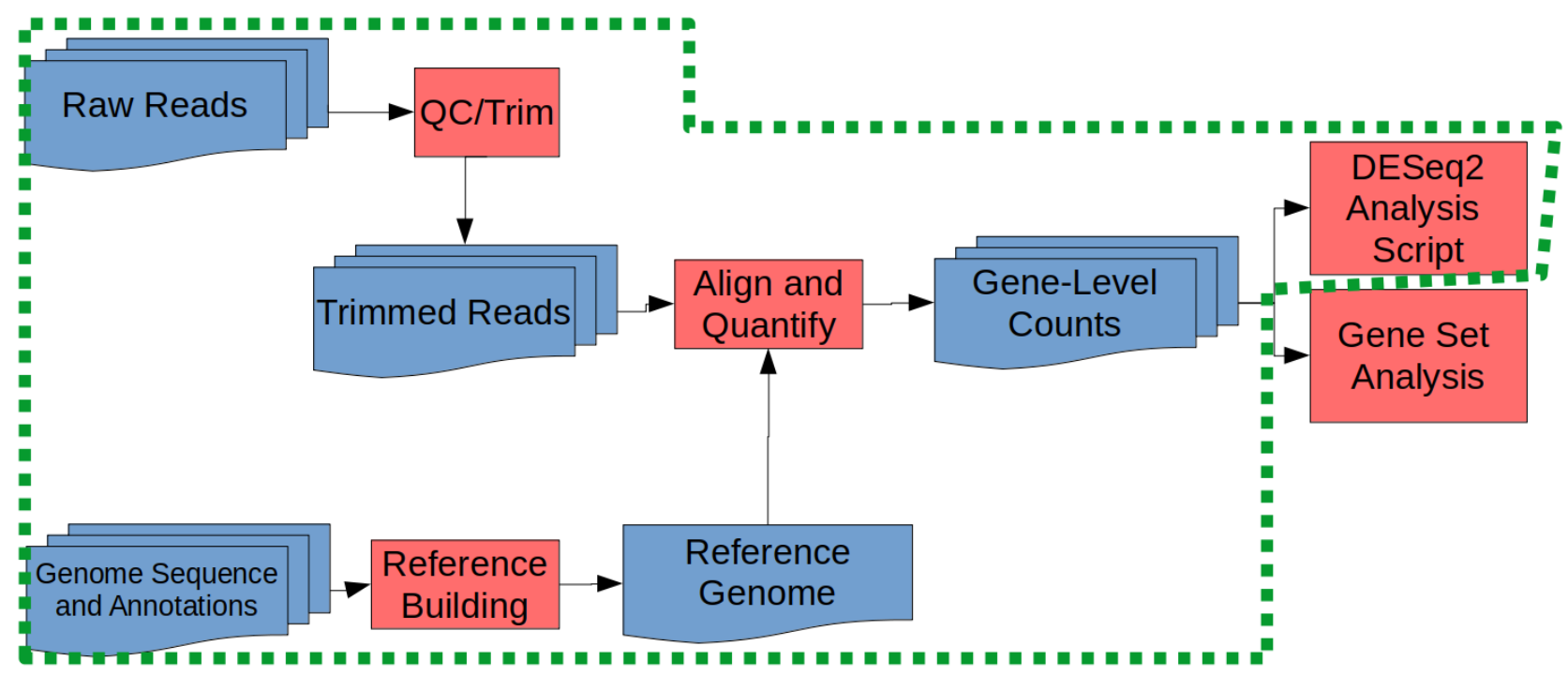

Figure 3. High Level Overview of Analysis Pipeline.

Files depicted by blue objects, processes depicted as red rectangles. Green dashed outline encloses RCP implemented in Nextflow.

\section{B. RCP Protocol}

\section{1) Quality Control and Trimming}

FastQC version 0.11 .9 was used to generate initial and trimmed quality metrics. Trim Galore version 0.6.4 and Cutadapt version 2.6 were used for adaptor removal [34]. In accordance with the RCP, neither deduplication nor quality trimming were performed [34]. MultiQC version 1.9 was used to inspect quality control results for all samples [35].

\section{2) STAR and RSEM Reference Building}

Mouse genome fasta (Mus_musculus.GRCm38.dna.toplevel.fa) and gtf annotation (Mus_musculus.GRCm38.96.gtf) files were obtained from ENSEMBL release 96. This was the same ENSEMBL release used in the GeneLab protocol, allowing more direct comparison of between GeneLab's posted results and the Nextflow implementation. References were built using both STAR version $2.7 .5 \mathrm{c}$ and RSEM version 1.3.3 [36]-[38]

\section{3) Alignment and Gene Count Quantification}

After adaptor removal, reads were mapped to the mouse genome using the splice aware mapping tool, STAR version 2.7.5c. Mapped reads were quantified using RSEM version 1.3.3 to generate an unnormalized counts table. MultiQC version 1.9 was used to inspect alignment results for all samples [35].

\section{4) Normalization and Differential Gene Expression Analysis Using Adapted DESeq2 Script}

A DESeq2 R script was obtained from Dr. Saravia-Butler and adapted to process GLDS- 104. Unnormalized count tables for every sample were read into $\mathrm{R}$ version 4.0.2. The script then uses DESeq2 version 1.28.1 for normalization and differential gene expression analysis. It is important to note that DESeq2 calculations assume gene counts follow a negative binomial distribution, which dictates the analysis approach.

The steps for the normalization and differential gene expression analysis are summarized as follows: First, genes were filtered out if their total count across all 12 samples was less than 10. Second, gene counts with excessive Cook's distances for a particular sample are marked as outliers and removed (note: this behavior is dependent on the number of samples per condition. If more than 6 samples per condition were in this experiment outliers would be replaced with imputed values rather than removed). 
Remaining counts were normalized for sample library size using the median-of-ratios method in DESeq2 [31]. This method involves calculating a sample size factor by computing the ratio of the gene count for a particular gene and sample over a pseudo-reference for the gene. The pseudo-reference for a gene is the geometric mean of the count across every sample for that gene. Next, once the ratios are computed for every gene and sample, the median of the ratios across each sample is taken as the size factor for that sample. Finally, each gene count is multiplied by the sample size factor, generating a normalized count ready for comparison across samples of varying library size.

After normalization, DESeq2 estimates the dispersion of each sample. This process can be summarized in three steps and is depicted in Fig. 4. First, gene-wise dispersion is initially estimated via maximum likelihood estimation (MLE). Next, using all genes' MLE dispersions and normalized count means, a smooth curve is fit to serve as a prior mean. Finally, gene MLE dispersions are brought closer to the dispersion estimated in the smooth curve using a maximum a posteri (MAP) approach. This results in a final estimated dispersion that is effectively interpolated between the initial gene-wise MLE dispersion and the gene-wise normalized count means vs MLE dispersions curve. Note, genes with MLE dispersions two standard deviations or greater away from the prior mean are not brought closer to the prior mean to avoid overcorrecting the dispersion estimate. For these genes, the MLE dispersion is used as the final dispersion estimate instead.

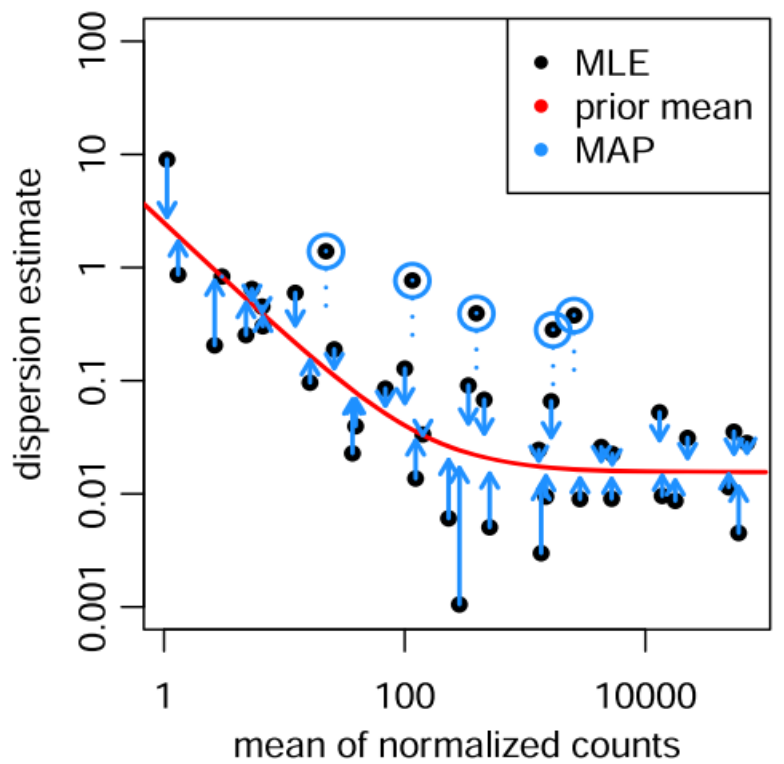

Figure 4. DESeq2 Dispersion Estimation Process For Subsampled Example Dataset Figure adapted from Huber et al [31]. Black points depict gene-wise MLE dispersions. Red curve is fit using all genes. Blue arrows indicate the shift to final dispersion estimate after MAP estimation using the red curve as the prior mean. Black points circled by blue circles are greater than two standard deviations away from prior mean and gene-wise MLE is taken as final dispersion instead of MAP estimation.

Gene expression log fold changes are computed and are shrunken towards zero to correct for dispersion's dependence on mean counts. This is described as trading high variance at the cost of bias towards smaller log fold changes. This correction is more significant for genes with smaller mean counts and higher final estimated dispersions. This shrunken log fold change is ultimately used to calculate statistical significance.

A Wald Test is performed to assess statistical significance. Specifically, the shrunken log fold change is taken as the test parameter and the null hypothesis assumes a normal distribution and consequently that the parameter is equal to zero - i.e., zero log fold change between space flight and ground control conditions. The shrunken log fold change is divided over the gene-wise standard error to yield a Z-statistic and associated p-value.

Once p-values have been calculated, an automatic independent filtering step is performed before 
calculating adjusted p-values using the Benjamini-Hochberg method. The filter is based on a minimum normalized mean count which is dynamically chosen by DESeq2 to optimize the significant genes detected at a specified false discovery rate (FDR). In the RCP script, this specified FDR is left at the default value of 0.1 ; however, this would be better set to the FDR threshold that will be used for final downstream analysis. Independent refers to the requirement the Wald Test Z-statistic distribution is "marginally independent" of the data used for filtering assuming the null hypothesis is true [31]. The DESeq2 authors argue this to be this the be the case; however, this project did not attempt to validate this argument [31].

P-values for genes that pass the filter undergo the Benjamini-Hochberg method to generate adjusted p-values that correspond to the FDR.

\section{Nextflow Implementation of RCP}

\section{1) Overview and Decision To Use Anaconda}

Nextflow version 20.07.01 and domain specific language 2 (DSL2) were chosen to take advantage of the modular process and simple workflow syntax. Version GL-DPPD-7101-C of the RCP documents every step and software used. These steps were converted into Nextflow processes and the processes were arranged into an overall workflow script, main.nf. While a Docker container based approach to pipeline software management would be more robust in terms of reproducibility, the COS-HPC currently does not support Docker and thus Anaconda was selected instead for managing software and associated dependencies. The full codebase is available at https://github.com/J-81/masterProject.

\section{2) Nextflow Processes}

Nextflow processes are encoded in module files as shown in Fig. 5. The ability to supply configuration parameters is demonstrated, here dictating the Anaconda environment file location. The "cpus" directive is determined by the number of reads utilized, two for the paired end read files in GLDS-104. The "cpus" directive is defined in the execution environment configuration files for cpu intensive processes. On the COS-HPC, each compute node has 28 cpus and this was the amount allocated to processes that could efficiently use the computational power. Nextflow Tower monitoring was used to assess the CPU efficiency of each process.

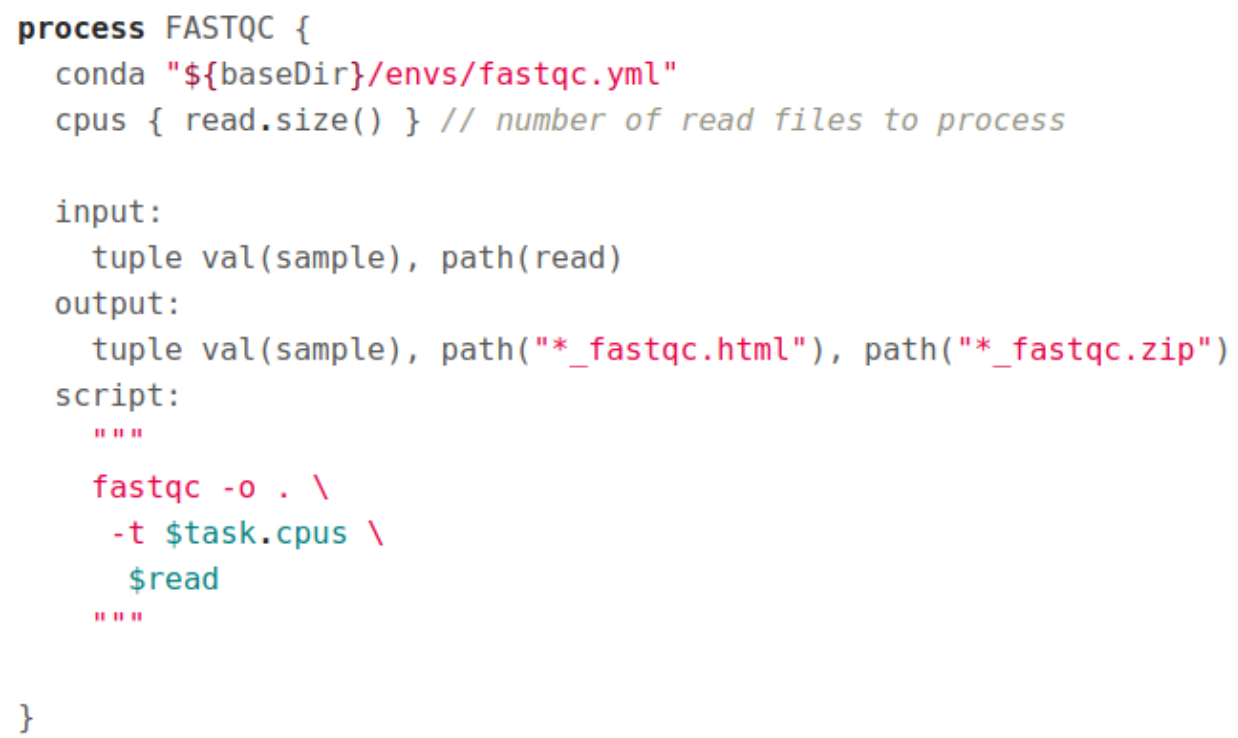

Figure 5. Nextflow Process For FASTQC.

3) Nextflow Workflow File

The workflow file imports the processes from modules, loads input files like raw reads as speci- 
fied in configuration files, and defines the connections between each process. These connections are defined as channels and channel modifying operators in Nextflow. Fig. 6 depicts the start of the workflow definition portion of the workflow file. Note, the ability to include conditional branches to allow the workflow flexibility. The if-else branch shown in Fig. 6 allows users to either supply read files by specifying their paths in the configuration file or retrieve the files directly from the GeneLab repository using the GET_DATA process.

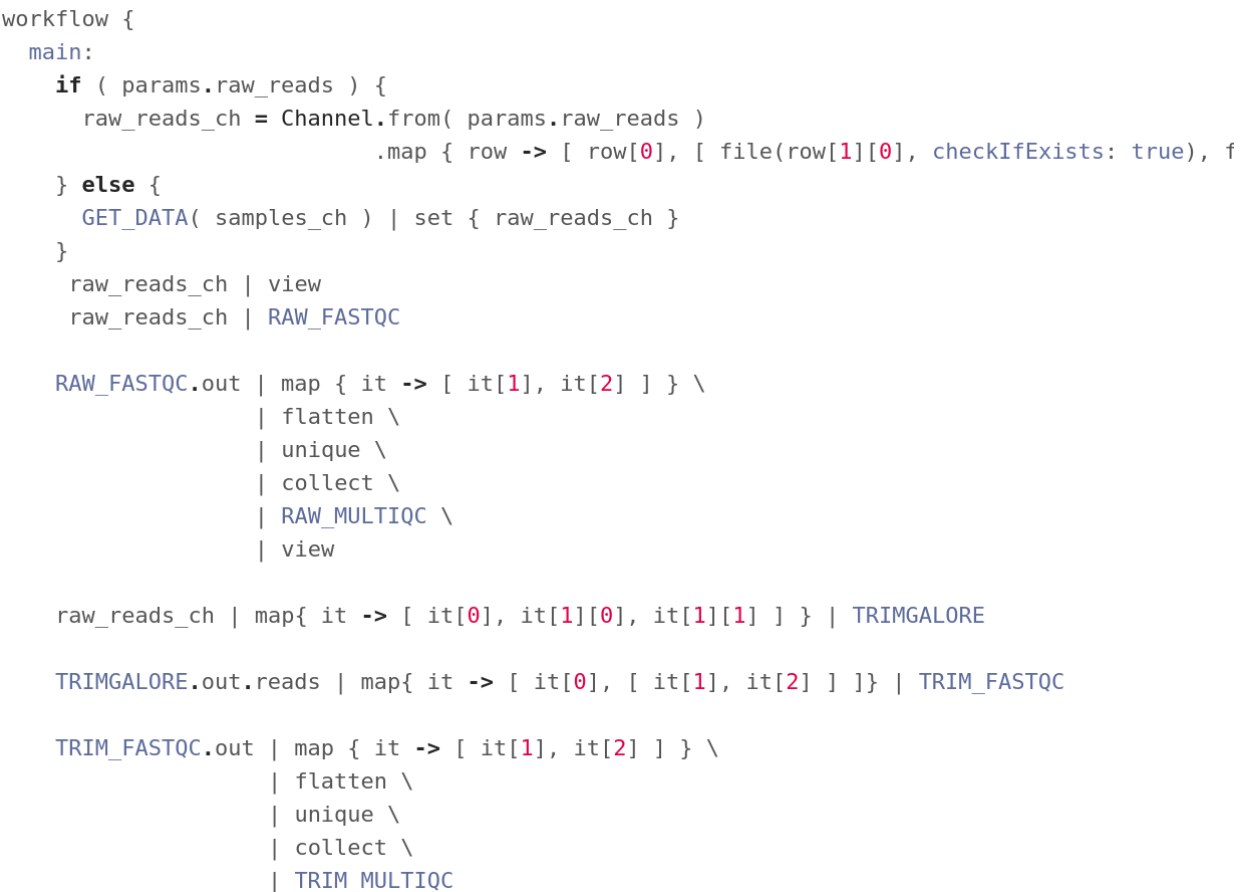

Figure 6. Nextflow Workflow File Excerpt

\section{4) Nextflow Configuration File}

Configuration files were created for two major aspects of the workflow. Fig. 7 depicts the entire configuration file for GLDS-104. This configuration file specifies the dataset to use for the workflow. This focuses on what data to operate on and is independent of specifying the execution environment.

Fig. 8 depicts the entire configuration file for running workflows on the COS-HPC. This defines execution environment parameters. Here processes can be assigned computational resources e.g. (CPU counts, RAM amounts, time limits) either by label or by process name that are appropriate to the process and execution environment. For example, as shown in Fig. 9, network bound processes tagged with the "networkBound" label are limited to a single process a time. This is because network bound processes are limited by the download speed of the execution environment and are not expected to benefit from parallel execution. Also note, in this execution configuration, jobs that do not require significant time or resources are allowed to run using the resources allocated for the Nextflow process itself rather than submitting new Slurm jobs. This preventing launching short duration and resource light processes that do not merit the overhead of their own Slurm job submission. 


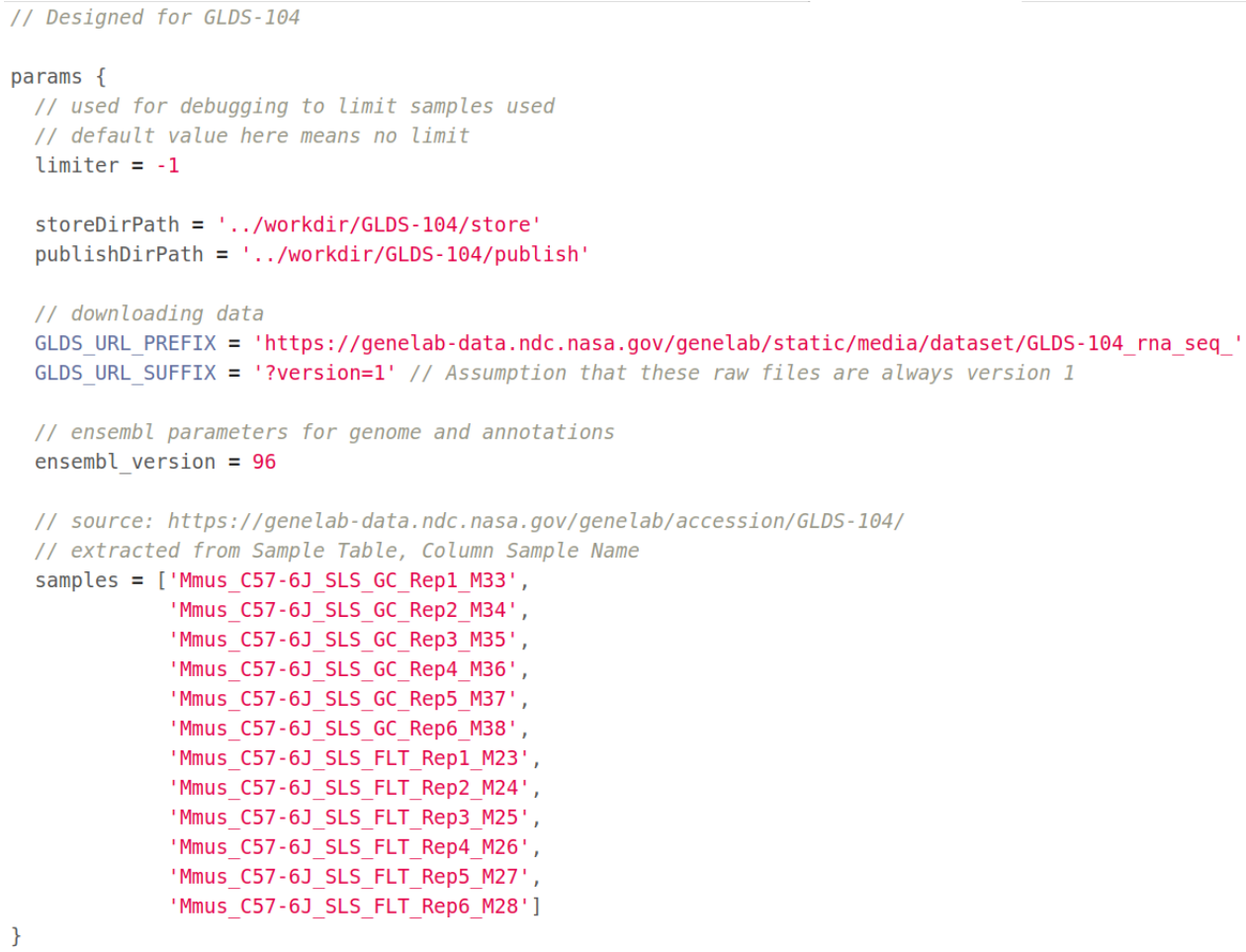

Figure 7. Workflow Configuration File.

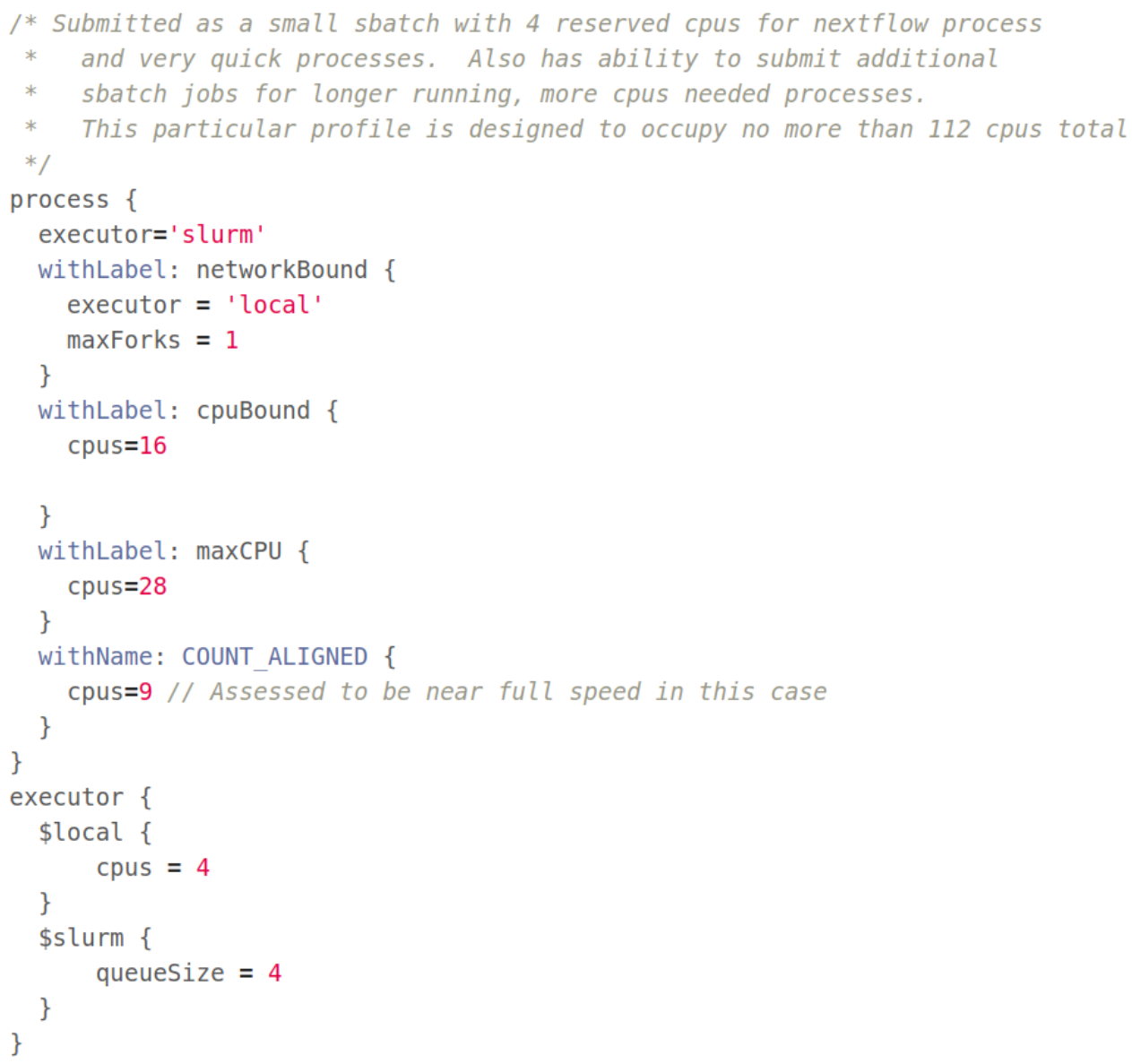

Figure 8. COS-HPC Executor Configuration File.

\section{5) Running The Workflow}

The workflow can be run on any Linux based system with Nextflow and Anaconda installed. 
The command to run the main workflow is as follows:

$>$ nextflow run $\mathrm{J}-81 /$ masterProject $-r$ main

This command will retrieve the workflow code directly from the GitHub repository and execute the workflow in a "work" directory created in the working directory where the command was issued. Configuration files are supplied using the "-profile" flag. Table I lists the current configuration files in the repository.

TABLE I

CONFIGURATION FILES

\begin{tabular}{cc}
\hline Configuration File Name & Description \\
\hline default.config & Directs workflow to use data from GLDS-104. \\
standard.config & Minimal configuration for local execution. \\
cos-hpc-4-node.config & Configuration for running on COS-HPC, utilizing Slurm for resource in- \\
& tensive jobs. \\
\hline
\end{tabular}

\section{6) Monitoring The Workflow With Nextflow Tower}

The workflow execution status was monitored using Nextflow Tower at https://tower.nf/ after creating an API token and exporting it as the "TOWER_ACCESS_TOKEN" environment variable. Information from Nextflow Tower was also used to refine resource allocation by process and assess aggregate resources used by the full pipeline.

\section{Extended DESeq2 Analysis Notebook}

An interactive analysis was performed using Jupyter Notebooks. Gene expression data was visualized using an MA plot and PCA plots generated by DESeq2 version 1.30.0. The Sankey diagram was generated using the python library, Plotly version 4.9.0. PCA plots were generated with both $\log _{2}$ (normalized counts) and the DESeq2 variance stabilizing transformation (VST) of the normalized counts. DESeq2's VST corrects for the dependence of variance on mean and is advised for visualizations that rely on the standardization to compare across different genes [39].

\section{E. Gene Set Analysis}

Analyzing significant genes individually can spotlight effects of space flight; however, given the complex relationship of many biological functions and sets of genes, a gene set approach to analysis can help find patterns of perturbations and describe them in terms of annotations curated by experts. Gene set analysis using annotation databases like the gene ontology database can enable comparisons between studies at a more complex level than individual genes alone [40].

\section{1) DAVID Gene Set Clustering}

The web tool for DAVID version 6.8 was used to find annotated gene sets enriched for significant genes after inputting a list of 3000 genes with the lowest adjusted p-values (all less than 0.05). This subset was selected as DAVID limits analysis to 3000 genes [32]. The tool was then used to generate gene set clusters based on the enriched gene sets with overlapping significant genes. Such clusters provide insight into sets of annotations that share a common theme.

\section{2) Preranked Gene Set Enrichment Analysis}

Gene set enrichment analysis was performed using GSEA version 4.1.0. The preranked method was employed using the Z-statistic output by DESeq2. The hallmark gene set collection from the MsigDB was used by first creating a chip2chip file mapping IDs between the hallmark gene set collection 
version 7.2 (Entrez IDs) and ENSEMBL gene IDS. Preranked GSEA was also performed using the gene ontology biological processes (GO BP) collection version 7.2 [33].

\section{3) Leading Edge Analysis On GO BP Gene Sets}

Leading Edge Analysis was performed using GSEA version 4.1.0. Preranked GSEA results against the GO BP collection were filtered down to 611 gene sets with nominal p-values $<0.05$ and submitted for Leading Edge Analysis.

\section{4) Cytoscape Enrichment Map Of GO Biological Processes}

Using the Enrichment Map plugin, Cytoscape version 3.8.2 was used to visualize the gene set overlaps and enrichment scores for significant gene sets[41] [42]. Gene sets were included in the enrichment map visualization if they met the following criteria: p-value $<0.005$, FDR $<0.1$, connected to at least 2 other gene sets. The p-value and FDR cutoffs are the default values for generating the enrichment map, considered between "moderately permissive" and "moderately conservative" in the Enrichment Map manual. Such settings are reasonable in the context of visualizing thematic networks composed of related gene sets [42]. Groups were manually annotated after arrangement using Cytoscape's circular layout.

\section{RESULTS}

\section{A. Nextflow Implementation Of RCP on GLDS-104}

\section{1) Comparison To GeneLab Posted Results For GLDS-104}

The final output table from DESeq2 retrieved from GeneLab's GLDS-104 repository was compared to the final output table from DESeq2 generated by this project's Nextflow RCP implementation. Fig. 9 depicts differences found between the two output files. The vast majority of significantly differentially expressed genes are identified in common between both the Nextflow RCP implementation and the GeneLab posted GLDS-104 results. Significantly differentially expressed genes unique to Nextflow are listed in Table. II. Likewise, significantly differentially expressed genes unique to GeneLab's posted results are listed in Table. III.
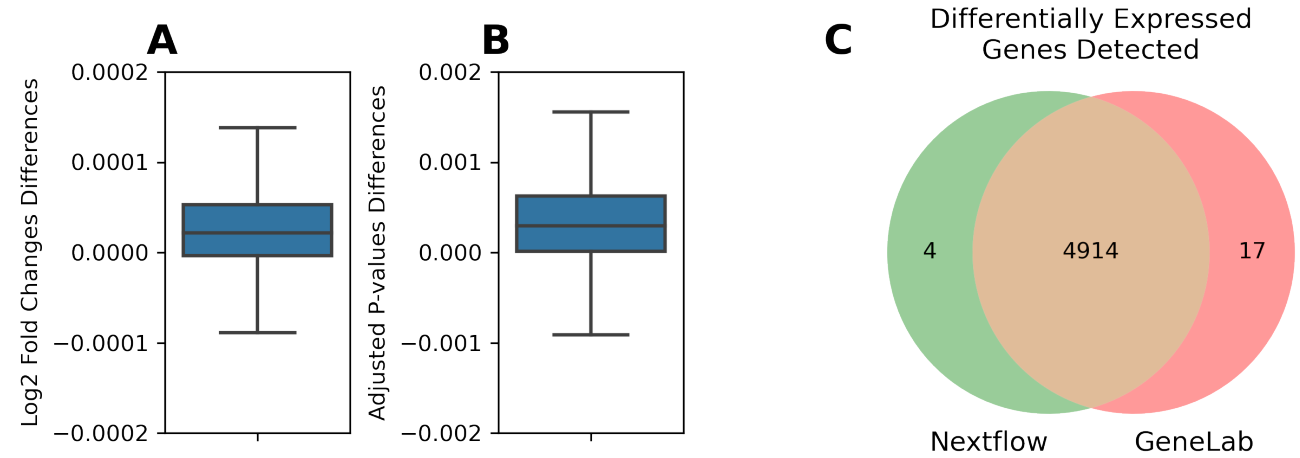

Figure 9. Nextflow Implementation VS GeneLab Posted Results (A) Boxplot of Log2(fold change) differences for all genes subjected to Wald Test (B) Boxplot of adjusted P-values differences for all genes subjected to Wald Test, adjusted P-value calculated by the Benjamini-Hochberg method. In both (A) and (B), outliers (defined as beyond $1.5 \mathrm{x}$ inner quartile range) are not shown. (C) Venn Diagram depicting shared and unique significantly differentially expressed genes identified in Nextflow and GeneLab Results. 
TABLE II

4 Genes Uniquely Detected As DifFerentially EXPRESSEd In NeXTFlow

\begin{tabular}{ccc}
\hline ENSEMBL ID & Description & Gene Name \\
\hline ENSMUSG00000078901 & predicted gene 14440 & Gm14440 \\
ENSMUSG00000082816 & predicted gene 11953 & Gm11953 \\
ENSMUSG00000049329 & predicted gene 15163 & Gm15163 \\
ENSMUSG00000106574 & predicted pseudogene 2451 & Gm2451 \\
\hline
\end{tabular}

TABLE III

17 Genes Uniquely Detected As Differentially ExPressed In GeneLab Posted Results

\begin{tabular}{ccc}
\hline ENSEMBL ID & Description & Gene Name \\
\hline ENSMUSG00000000325 & armadillo repeat gene deleted in velocardiofacial syndrome & Arvcf \\
ENSMUSG00000005262 & ubiquitin recognition factor in ER-associated degradation 1 & Ufd1 \\
ENSMUSG00000005625 & proteasome (prosome, macropain) 26S subunit, non-ATPase, 4 & Psmd4 \\
ENSMUSG00000017776 & v-crk avian sarcoma virus CT10 oncogene homolog & Crk \\
ENSMUSG00000020219 & translocase of inner mitochondrial membrane 13 & Timm13 \\
ENSMUSG00000024151 & mutS homolog 2 & Msh2 \\
ENSMUSG00000029178 & Kruppel-like factor 3 (basic) & Klf3 \\
ENSMUSG00000029267 & metal response element binding transcription factor 2 & Mtf2 \\
ENSMUSG00000032359 & cathepsin H & Ctsh \\
ENSMUSG00000053291 & RAB4B, member RAS oncogene family & Rab4b \\
ENSMUSG00000073530 & pappalysin 2 & Pappa2 \\
ENSMUSG00000078300 & predicted pseudogene 2606 & Gm2606 \\
ENSMUSG00000078965 & predicted gene 12033 & Gm12033 \\
ENSMUSG00000081600 & predicted gene 12286 & Gm12286 \\
ENSMUSG00000092131 & cDNA sequence BC050972 & BC050972 \\
ENSMUSG00000094388 & predicted pseudogene 8783 & Gm8783 \\
ENSMUSG00000096592 & predicted gene 15801 & Gm15801 \\
\hline
\end{tabular}

\section{2) RCP Computing Resource Requirements}

Key resources used on the COS-HPC for the Nextflow implementation of the RCP executed on GLDS-104 are shown in Table IV. Actual time required to complete the entire RCP, including downloading the initial data, was less than 24 hours. An insignificant amount of time $(<5$ minutes $)$ was spent waiting for the COS-HPC resources as excess resources were available when GLDS-104 was processed. Note GLDS-104's raw data is composed of $\sim 900$ million 100 base pair pair ended reads. 
TABLE IV

SUMMARY OF RESOURCES FOR RCP PERFORMED ON COS-HPC FOR GLDS-104

\begin{tabular}{cc}
\hline Resource & Description \\
\hline CPU Hours & 527 CPU Hours \\
Peak RAM & $52.41 \mathrm{~GB}$ \\
Peak Disk Memory Write & $307 \mathrm{~GB}$ \\
\hline
\end{tabular}

\section{B. Quality Control Metrics}
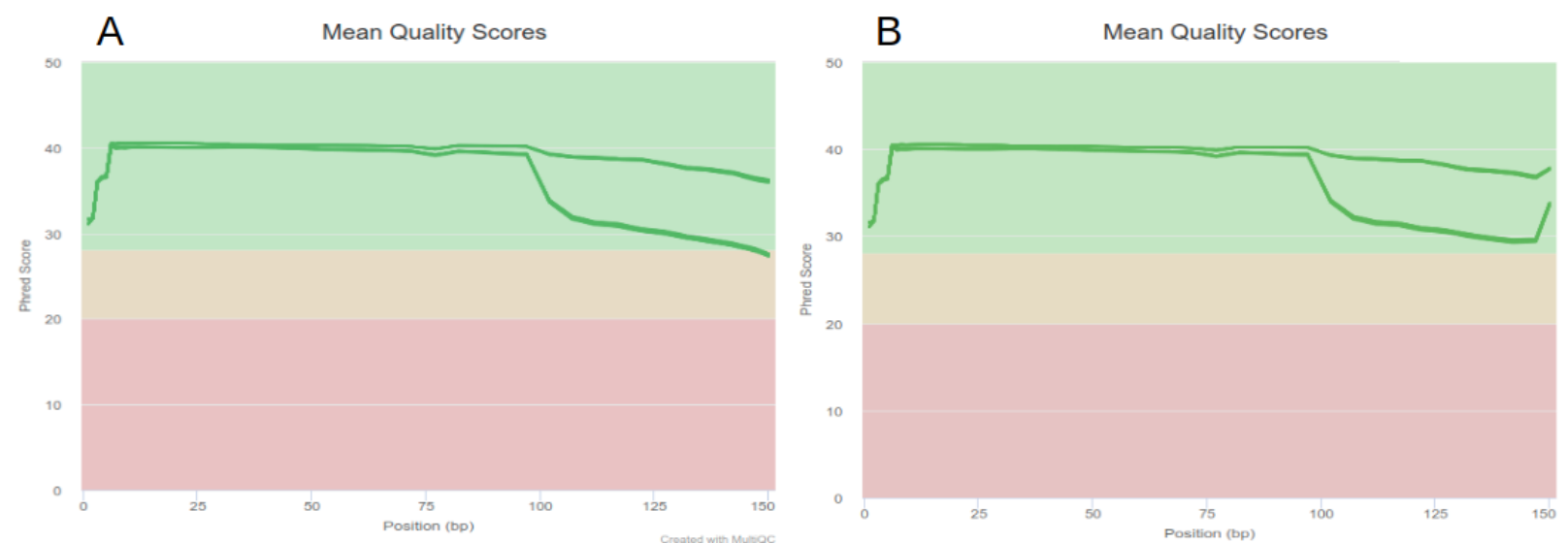

Figure 10. MultiQC Mean Quality Score Plots

(A) Untrimmed Reads (B) Trimmed Reads

The MultiQC report indicated results largely normal for RNA-Seq experiments. The mean quality scores shown in Fig. 10 indicate these reads were accurate throughout. Post trim step and adapter removal, the quality even improves slightly at the last base pairs. Another feature recognized is the drop in quality for reverse reads. Reverse reads do tend to be worse. Even then, these reverse reads are of high quality based on their Phred scores. MultiQC also reported adapter contamination was successfully controlled after the trimming step with no samples containing greater than $0.1 \%$ adapter contamination. There was a detected nucleotide composition bias in the initial 7 base pairs.

\section{Difference In Requested Read Lengths And Observed Read Lengths}

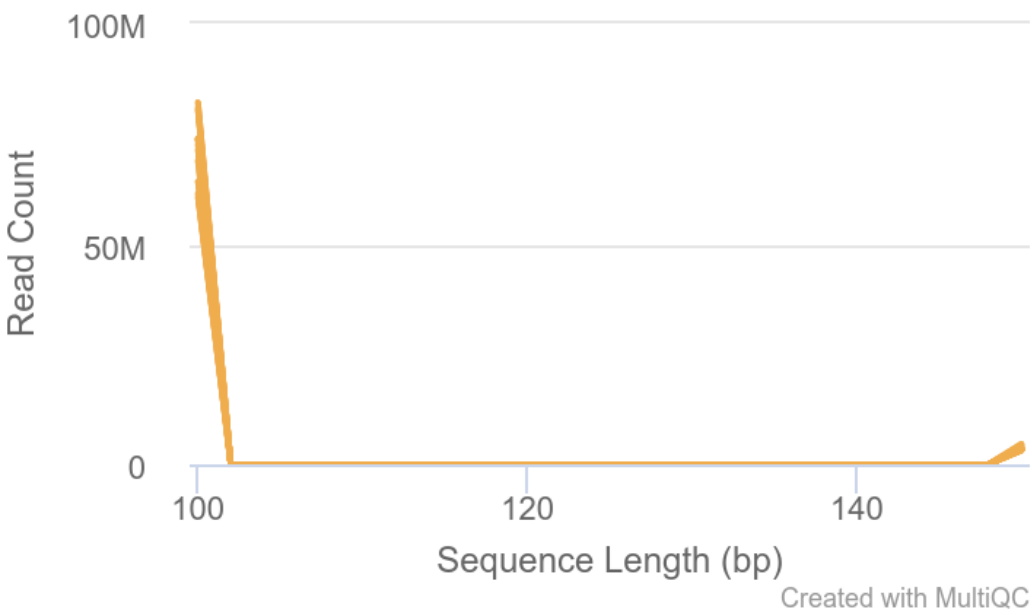

Figure 11. Sequence Length Distribution 
Sequence length distribution in Fig. 11 shows that most reads are consistent with 100 base pair read length sequencing described by GLDS-104 protocol; however, there exists a small subset of reads with lengths of 150 base pairs.

\section{Alignment Metrics}

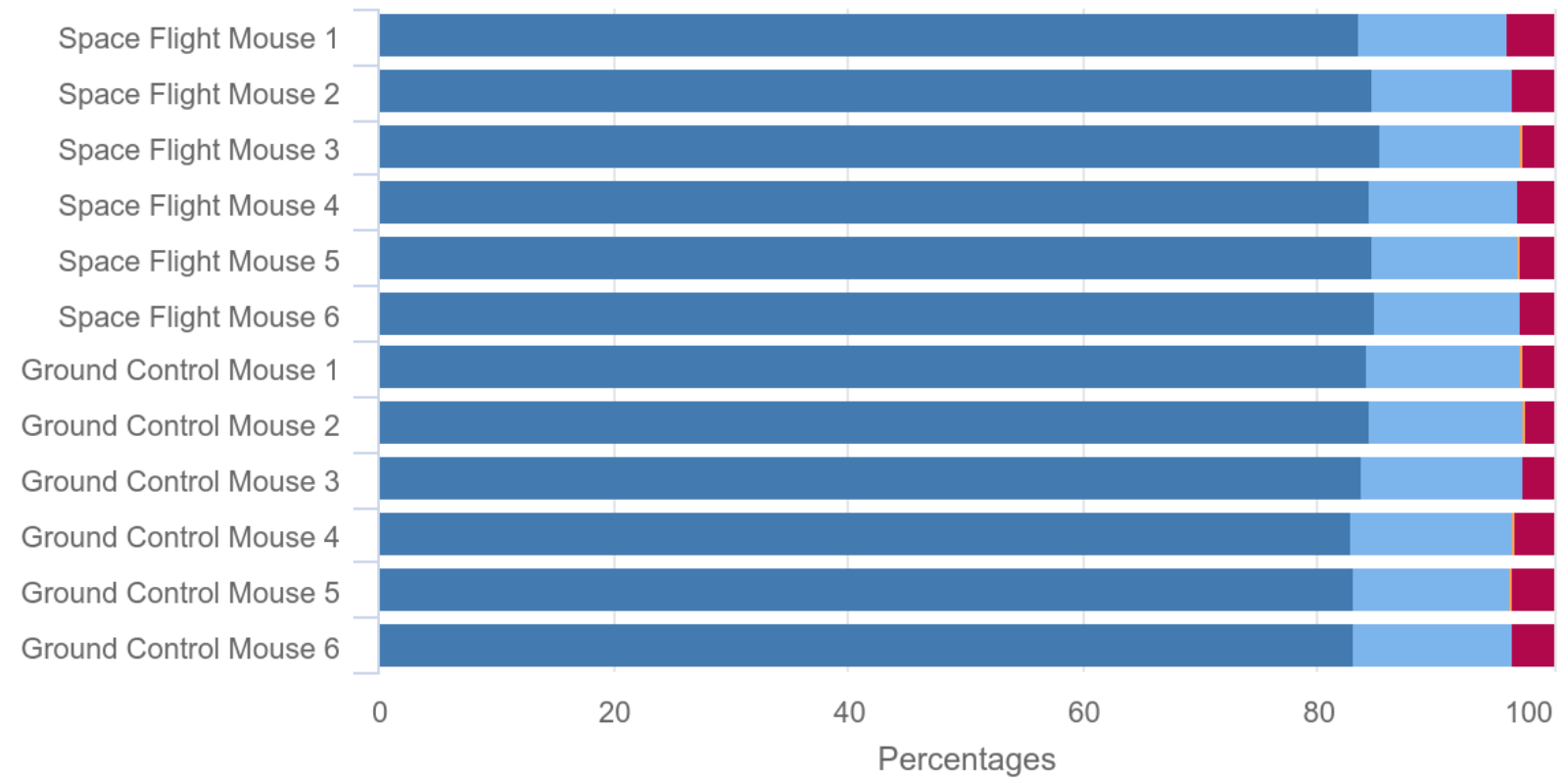

Uniquely mapped Unmapped: too short
Mapped to multiple loci

Unmapped: other
Mapped to too many loci

Created with MultiQC

Figure 12. Star Alignment Metrics

Fig. 12 shows the mapping results for each sample. At least $82.8 \%$ of reads for every sample were uniquely mapped to a location. Approximately $12.5 \%$ of reads were mapped to 2-10 loci. Approximately $3 \%$ of each sample were left unmapped due to short alignment length. A very small amount of the reads (less than $0.2 \%$ ) were either mapped to too many loci (over 10 ) or unmapped for other reasons. 
E. Gene Counting Metrics

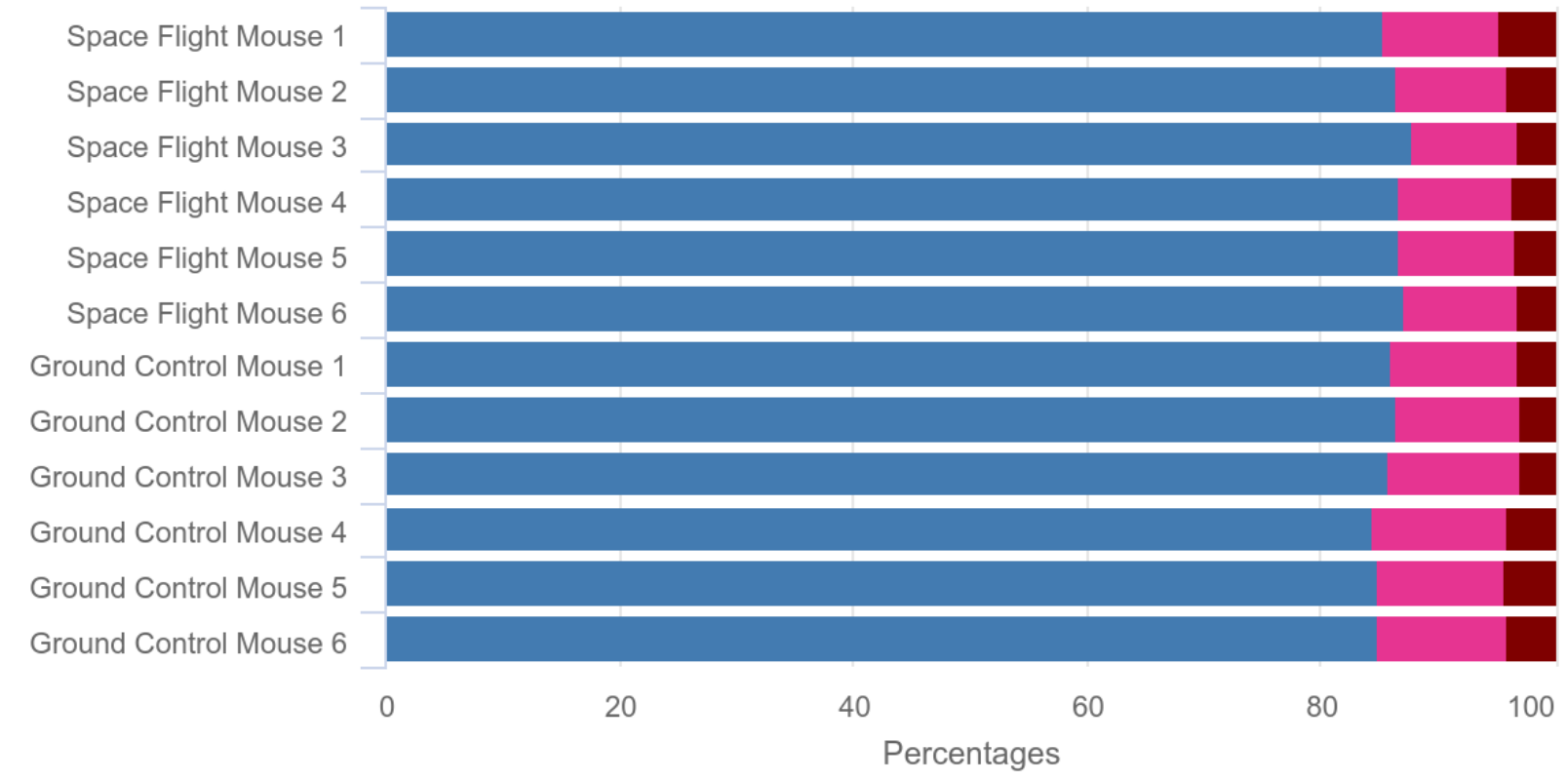

Aligned uniquely to a gene Aligned to multiple genes

Filtered due to too many alignments Unalignable reads

Created with MultiQC

Figure 13. RSEM Counting Results

At least $84.8 \%$ of the reads per sample were uniquely mapped to a gene. Approximately $10 \%$ of reads were aligned to multiple genes (although no more than 10). Zero reads were filtered out due to too many alignments (greater than 10). Approximately 4\% of reads could not be aligned to gene features.

F. Gene Level Differential Expression Using DESeq2

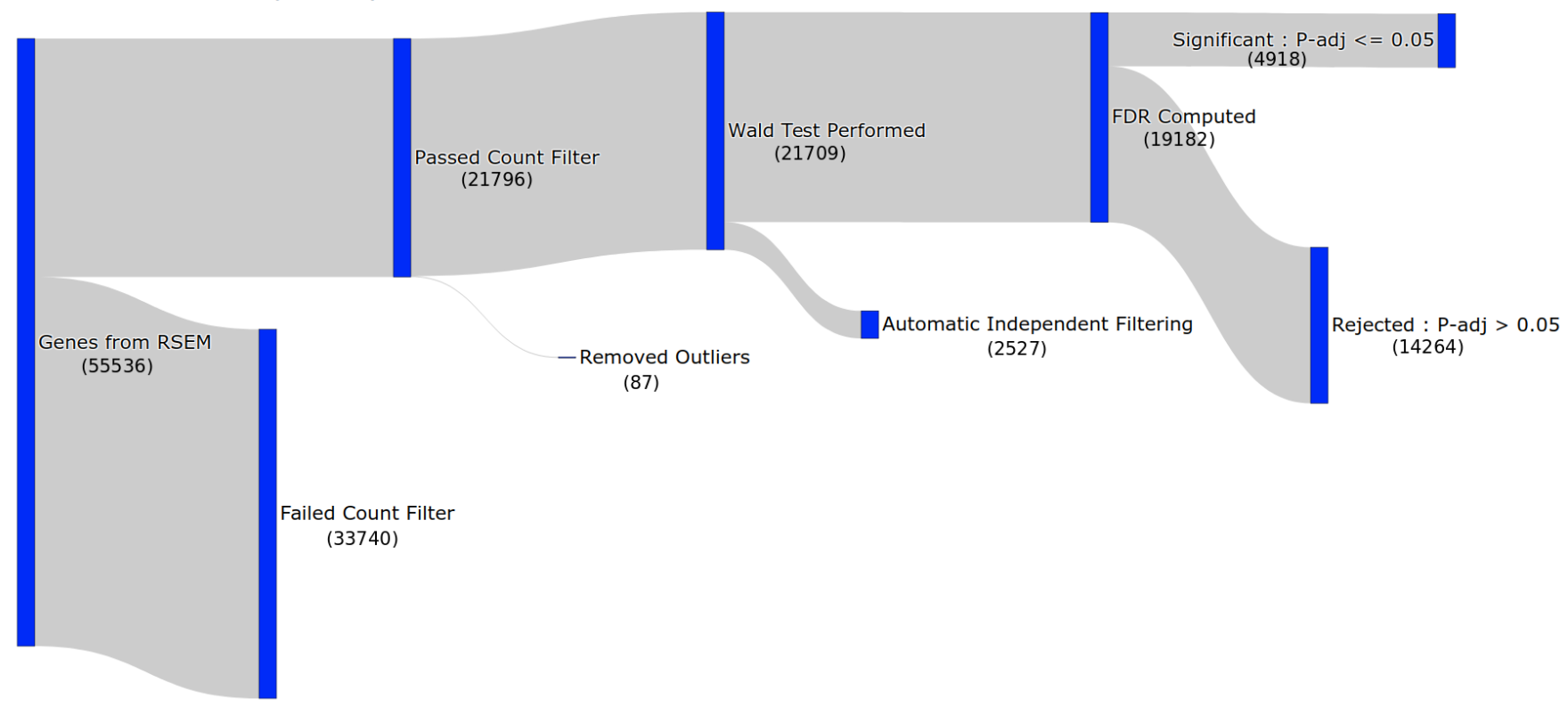

Figure 14. Sankey Diagram For Genes Processed Through DESeq2

Number of genes for each step shown in parentheses. 


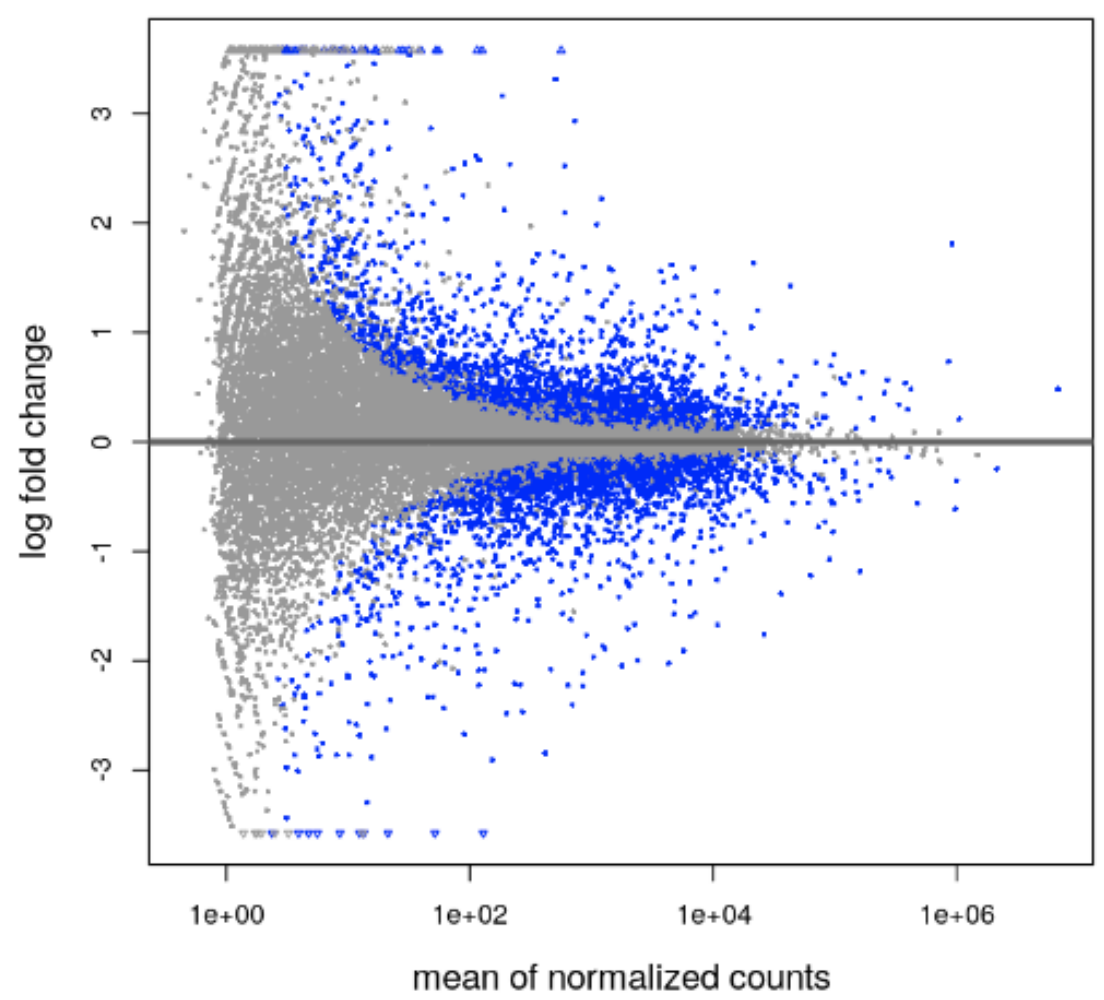

Figure 15. MA Plot

Each point represents a single gene. Points depicted in blue are significant (Benjamini-Hochberg method derived adjusted $p$-value $<0.05$ )
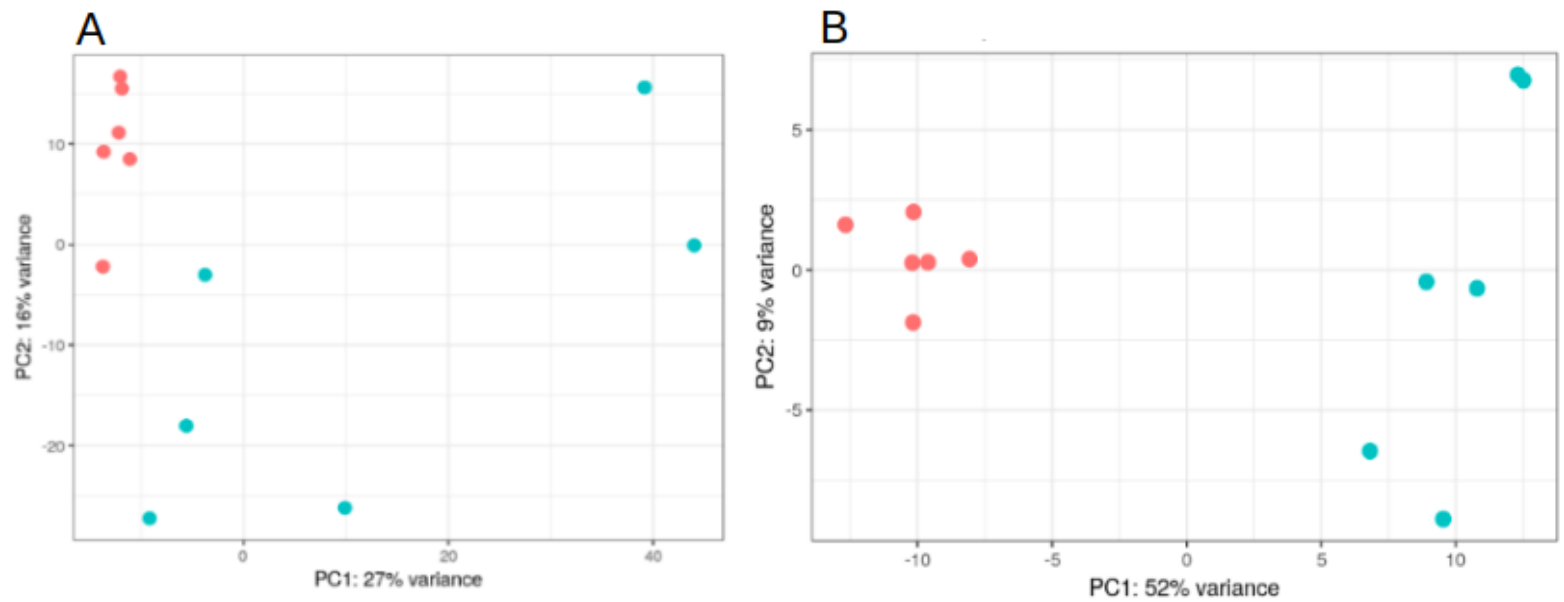

Figure 16. Principal Component Analysis Plots For Samples

(A) PCA using $\log _{2}$ (Normalized Counts) (B) PCA using normalized counts after variance stabilized transformation. Space flight samples depicted in blue. Ground control samples depicted in red.

The Sankey diagram in Fig. 14 shows how genes were culled at each step in the DESeq2 script. The number of outliers is minimal further suggesting no significant abnormalities were detected before statistical testing was performed.

A total of 4918 genes were found to be significantly differentially expressed when controlling for an FDR $<0.05$. Fig. 15 depicts an MA plot of all genes and does not suggest any abnormalities in analysis. The PCA plot presented in Fig. 16 (A) does not appear to differentiate ground control and space flight samples. The amount of variation included in graphed PC1 and PC2 is low suggesting that additional variation no shown may explain the inability to differentiate between treatment groups. Upon re- 
plotting using DESeq2's VST, suggested for visualizations, the PCA plot in Fig. 16 (B) better demonstrates a distinct difference between treatment groups. Given the large number of significant genes, the impact of space flight on gene expression is widespread.

\section{G. Gene Set Analysis}

\section{1) DAVID Gene Set Clusters}

Two of the top three significant annotation clusters in Table V suggest disruptions of the endoplasmic reticulum and sarcoplasmic reticulum related gene expression. The sarcoplasmic reticulum is an organelle differentiated from the endoplasmic reticulum. These are concentrated near myofibrils and play an important role muscle contraction mediated by controlling local $\mathrm{Ca}+$ concentration[43]. In addition to metal binding proteins as the second leading annotation cluster, this suggests a more specific effect of space flight on the function of sarcoplasmic reticulum in overall muscle contraction/extension. In Table VI, a search of PubMed against these key terms suggests that the connection between space flight and sarcoplasmic reticulum are less studied than the more generalized effect on endoplasmic reticulum. "Metal binding" searches did not yield nearly as many hits; however, this was the most general of the three search terms. Potentially relevant articles may have also been missed if they used generally synonymous terms including "metal ion binding", "cation binding", and "cation ligands".

TABLE V.

TOP THREE ENRICHED ANNOTATION DAVID CLUSTERS

\begin{tabular}{ccc}
\hline Category & Term & FDR \\
\hline Cluster 1 & Endoplasmic reticulum & $1.5 \mathrm{E}-12$ \\
UP_KEYWORDS & GO:0005783 endoplasmic reticulum & $4.2 \mathrm{E}-08$ \\
GOTERM_CC_DIRECT & GO:0005789 endoplasmic reticulum & $2.8 \mathrm{E}-02$ \\
GOTERM_CC_DIRECT & membrane & \\
Cluster 2 & Metal-binding & $1.3 \mathrm{E}-14$ \\
UP_KEYWORDS & GO:0046872 metal ion binding & $2.1 \mathrm{E}-06$ \\
GOTERM_MF_DIRECT & Zinc & $9.4 \mathrm{E}-07$ \\
UP_KEYWORDS & GO:0008270 zinc ion binding & $3.6 \mathrm{E}-04$ \\
GOTERM_MF_DIRECT & Zinc-finger & $1.1 \mathrm{E}-03$ \\
UP_KEYWORDS & GO:0016529 sarcoplasmic reticulum & \\
Cluster 3 & Sarcoplasmic reticulum & $4.2 \mathrm{E}-08$ \\
GOTERM_CC_DIRECT & GO:0033017 sarcoplasmic reticulum & $1.2 \mathrm{E}-06$ \\
UP_KEYWORDS & membrane & $9.6 \mathrm{E}-04$ \\
GOTERM_CC_DIRECT & & \\
\hline
\end{tabular}


TABLE VI.

PubMed Query For Key Terms From Top DAVID Clusters

\begin{tabular}{lc}
\hline \multicolumn{1}{c}{ PubMed Query Term } & Number of Hits \\
\hline "Endoplasmic reticulum" microgravity & 50 \\
"Endoplasmic reticulum" "space" radiation & 71 \\
"Metal binding" microgravity & 1 \\
"Metal binding" "space" radiation & 4 \\
"sarcoplasmic reticulum" microgravity & 29 \\
"sarcoplasmic reticulum" "space" radiation & 14 \\
\hline
\end{tabular}

\section{2) Gene Set Enrichment Analysis}

The three significant hallmark gene sets enriched in upregulated genes after GSEA when controlling for FDR $<0.25$ are presented in Table VII. This less stringent threshold for FDR compared to the gene level analysis was recommended by GSEA and is reasonable given the number of gene sets tested in the hallmark gene set, 30, is much smaller than the number of genes tested in differential expression analysis. Additionally, Table VIII presents hallmark gene sets enriched for downregulated genes. Gene set enrichment results using the hallmark gene sets were exactly the same when removing the 4 genes unique to the Nextflow results and including the 17 genes unique to the GeneLab posted results.

Leading edge analysis results are presented in Table IX and indicates genes that were the most prevalent among GO BP gene sets. These genes are connected to many significant GO BP annotations indicating roles in numerous biological processes.

The enrichment map of GO BP gene sets presented in Fig. 17 groups GO BP annotations to demonstrate how the space flight condition has an widespread effect on different cellular components and related processes. When removing the 4 genes unique to the Nextflow results and including the 17 genes unique to the GeneLab posted results, minimal changes to significant GO BP gene sets were found ( 2 of the 1160 significant gene sets upregulated in space in Nextflow were found insignificant and 5 of 1158 insignificant gene sets downregulated in space in Nextflow were found significant).

TABLE VII.

GSEA Space Flight Upregulated Hallmark Gene Sets

\begin{tabular}{lcccc}
\hline \multicolumn{1}{c}{ NAME } & NES & NOM p-val & FDR & FWER p-val \\
\hline HALLMARK_MYOGENESIS & 1.75 & 0 & 0.14 & 0.18 \\
HALLMARK_KRAS_SIGNALING_DN & 1.7 & 0.01 & 0.09 & 0.23 \\
HALLMARK_HYPOXIA & 1.65 & 0.02 & 0.09 & 0.32 \\
\hline
\end{tabular}


TABLE VIII.

GSEA Space Flight Downregulated Hallmark Gene Sets

\begin{tabular}{lcccc}
\hline \multicolumn{1}{c}{ NAME } & NES & NOM p-val & FDR & FWER p-val \\
\hline HALLMARK_REACTIVE_OXYGEN_SPECIES_PATHWAY & -1.88 & 0.01 & 0.11 & 0.09 \\
HALLMARK_XENOBIOTIC_METABOLISM & -1.81 & 0 & 0.1 & 0.16 \\
HALLMARK_PROTEIN_SECRETION & -1.8 & 0.02 & 0.07 & 0.17 \\
HALLMARK_P53_PATHWAY & -1.8 & 0 & 0.05 & 0.17 \\
HALLMARK_UNFOLDED_PROTEIN_RESPONSE & -1.73 & 0.01 & 0.07 & 0.26 \\
HALLMARK_HEME_METABOLISM & -1.6 & 0.02 & 0.13 & 0.49 \\
HALLMARK_MYC_TARGETS_V2 & -1.58 & 0.06 & 0.14 & 0.56 \\
HALLMARK_FATTY_ACID_METABOLISM & -1.51 & 0.04 & 0.18 & 0.71 \\
HALLMARK_PI3K_AKT_MTOR_SIGNALING & -1.5 & 0.05 & 0.17 & 0.73 \\
HALLMARK_APOPTOSIS & -1.46 & 0.07 & 0.18 & 0.78 \\
HALLMARK_MYC_TARGETS_V1 & -1.42 & 0.06 & 0.21 & 0.85 \\
HALLMARK_TGF_BETA_SIGNALING & -1.41 & 0.1 & 0.21 & 0.88 \\
HALLMARK_ESTROGEN_RESPONSE_LATE & -1.39 & 0.1 & 0.21 & 0.9 \\
HALLMARK_MTORC1_SIGNALING & -1.37 & 0.09 & 0.22 & 0.92 \\
\hline
\end{tabular}


TABLE IX.

Prevalent Genes From Leading Edge Analysis On 611 GO BP Gene Sets

\begin{tabular}{|c|c|c|c|}
\hline ENSEMBL ID & Description & $\begin{array}{c}\text { Expression In Space Flight } \\
\text { Compared To Ground Con- } \\
\text { trol }\end{array}$ & $\begin{array}{l}\text { Times Present In Leading } \\
\text { Edge Of Gene Sets }\end{array}$ \\
\hline ENSMUSG00000032402 & SMAD family member 3 & Upregulated & 95 \\
\hline ENSMUSG00000018411 & $\begin{array}{l}\text { microtubule-associated pro- } \\
\text { tein tau }\end{array}$ & Downregulated & 95 \\
\hline ENSMUSG00000031980 & $\begin{array}{c}\text { angiotensinogen (serpin pep- } \\
\text { tidase inhibitor, clade A, } \\
\text { member } 8 \text { ) }\end{array}$ & Upregulated & 78 \\
\hline ENSMUSG00000031161 & histone deacetylase 6 & Downregulated & 77 \\
\hline ENSMUSG00000015656 & heat shock protein 8 & Downregulated & 76 \\
\hline ENSMUSG00000021270 & $\begin{array}{l}\text { heat shock protein } 90 \text {, alpha } \\
\text { (cytosolic), class A member } 1\end{array}$ & Downregulated & 72 \\
\hline ENSMUSG00000023944 & $\begin{array}{l}\text { heat shock protein } 90 \text {, alpha } \\
\text { (cytosolic), class B member } 1\end{array}$ & Downregulated & 72 \\
\hline ENSMUSG00000020122 & $\begin{array}{l}\text { epidermal growth factor re- } \\
\text { ceptor }\end{array}$ & Upregulated & 66 \\
\hline ENSMUSG00000032440 & $\begin{array}{l}\text { transforming growth factor, } \\
\text { beta receptor II }\end{array}$ & Upregulated & 66 \\
\hline ENSMUSG00000027254 & $\begin{array}{c}\text { microtubule-associated pro- } \\
\text { tein } 1 \mathrm{~A}\end{array}$ & Downregulated & 64 \\
\hline ENSMUSG00000052727 & $\begin{array}{c}\text { microtubule-associated pro- } \\
\text { tein } 1 \mathrm{~B}\end{array}$ & Downregulated & 63 \\
\hline
\end{tabular}




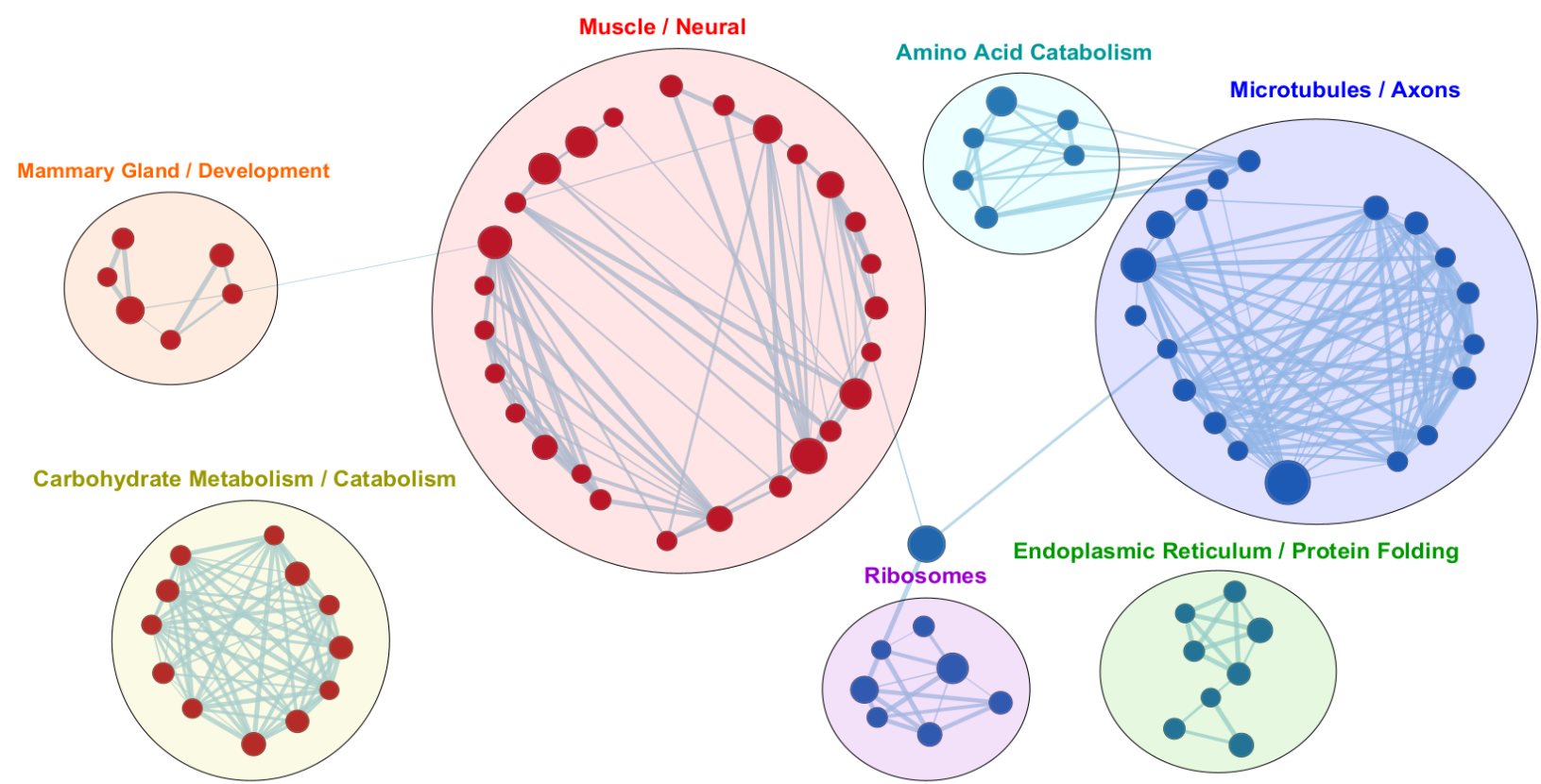

Figure 17. GO Biological Processes Collection Enrichment Map.

Each node represents a significantly upregulated or downregulated GO BP gene set. Red nodes indicate upregulation, blue indicate downregulation. Connections drawn depict overlap of significant genes in shared between gene sets.

\section{DISCUSSION}

\section{A. Nextflow Compared To Previous Implementations}

Before implementing the RCP using Nextflow, the RCP was implemented by Bash script and Snakemake. While both bash scripting and Snakemake were able to recreate the RCP, Nextflow was a more optimal solution due to a few key features. First, Nextflow includes a system for workflow versioning through tight integration with GitHub [29]. Second, Nextflow includes modular process definitions that allow one to reuse modules between different workflows. While Snakemake include an analogous feature, Nextflow's documentation was easier to follow and implement. Custom bash scripting could implement modularity; however, this was not explored in this project. Third, Nextflow has integration with Nextflow Tower, a free web based monitoring service. Nextflow Tower monitoring enabled me explore errors and optimize process resources based on reports of resource efficiency. These features make Nextflow the best choice for implementing the RCP among the three implementation options explored.

\section{B. Discussing Results For GLDS-104}

\section{1) Validation Of Nextflow Implementation}

Differences between results presented in Fig. 9 indicate that the Nextflow process did not produce exactly the same results; however, the majority of significant genes detected is mostly reproduced and deviations in both log fold change and adjusted p-values were minimal for most genes. These deviations did not produce any changes in the hallmark gene set analysis and minor changes in the GO BP gene set analysis.

For comparision, an investigation of reproducibility for a Kallisto and Sleuth based differential gene expression pipeline using Nextflow resulted in larger deviations in detected significant genes related to operating system differences [29]. The suggests the variation observed in this project compared to the posted GeneLab results for GLDS-104 may be due to operating system differences, non-deterministic outcomes related to cpu/thread count and variations in tool versions rather than a failure to correctly implement the RCP. Future work should be performed to determine the exact causes of the differences to 
better understand and address factors that reduce reproducibility. Additional results from the same study [29] indicate that a Docker based version of the Nextflow implementation may result in a more accurate reproduction of results. This further suggests that availability of Docker support for high performance compute environments may be necessary for more complete reproducibility of results across different compute environments.

\section{2) RCP Resource Requirements}

The total size of the merged raw read files for GLDS-104 that serve as the initial data for the RCP is over 95 gigabytes. Downloading this amount of data can be a large initial bottleneck. This suggests environments with a high download speed are necessary for these kinds of analyses. Another potential bottleneck is indicated by the aggregate CPU hours in Table IV. The RCP data processing and analysis required over $500 \mathrm{CPU}$ hours. This would suggest a multi-day or even multi-week runtime on a conventional computer. On the COS-HPC, processes are given scaled up resources and run in parallel where appropriate. With these improvements, the RCP performed on the COS-HPC can be completed in a single day provided there are sufficient resources available.

A high amount of RAM is also required for certain portions of the RCP. The maximum RAM required, 52.41 gigabytes, is due to STAR reference building. This is may represent another bottleneck in cases where a new organism or ENSEMBL release need to be processed. It is important to note that the once built, an organism reference can be reused with other RNA-Seq datasets reducing the computational load.

The final resource requirement to be considered is peak disk write size of over 300 gigabytes achieved by the trimming step. The read/write speed to disk can be considered another bottleneck during such steps. On the COS-HPC, this bottleneck was addressed by executing the process on the high read/ write speed "scratch" space. Altogether these practical considerations based on the RCP performed on GLDS-104 suggest that completing such analysis in a reasonable time requires access to high performance computing and efficient workflow configuration.

\section{3) Quality Control}

While quality trimming may be an option to remove lower quality reads or portions of reads, the RCP does not perform quality trimming. The preprint for the RCP notes this was avoided as Williams et al. demonstrated quality trimming negatively impacts expression quantification [27], [44] .

Another interesting observation was the disconnect between the 100 base pair read length noted in the GLDS-104 protocol and the actual read length distribution shown in Fig. 11 [30]. This suggests a different 150 base pair read length protocol was also employed and pooled with the 100 base pair read length data; however, the protocol does not describe such a procedure nor was I able to determine why 150 base pair and 100 base pair data would be pooled. Further evidence that a different 150 base pair sequencing protocol was employed in generating this data is a distinct drop off in quality for positions 101150 shown in Fig. 10. This suggests average quality positions 101-105 may be attributed to a separate sequencing protocol with its own average quality. Future investigation into this anomaly would include plotting average quality by position for 100 base pair reads and 150 base pair reads separately.

Further quality control measures should be used to extend the RCP. First, FastQC is unlikely to find issues associated with sample degradation. RSeQC is a program that uses raw read and gene annotation information to detect possible mRNA degradation in the sample [45] . Another method for extending quality control past the raw reads step would be to implement scripts for validating the output for each step of the RCP.

\section{4) Read Alignment and Expression Quantification}

Read alignment statistics presented in Fig. 12 demonstrate the vast majority of reads were successfully mapped to either unique locations or multiple locations. A small portion resulted in alignments that were too short. to map. An experiment with longer read lengths should reduce the amount unmapped 
due to insufficient alignment length. Longer reads would likely also reduce the amount mapped to multiple loci as longer reads present an opportunity for longer alignments and thus less potential for ambiguity.

Gene alignment statistics for STAR alignments converted to gene alignments through RSEM are presented in Fig. 13. Similarly, these results show the vast majority of reads map to unique genes. Again, longer read lengths would reduce the portion mapping to multiple genes by reducing ambiguous alignments. Interestingly, a significant portion of reads, 4\%, were marked as unalignable. This may be due in part to differences in mapping strategies between RSEM and STAR and/or the anomalous inconsistency in read length although this project did not investigate this further.

\section{5) Differential Gene Expression}

The inclusion of a number of heuristic methods in DESeq2 seems reasonable but an extended analysis comparing results with and without the automatic independent filtering step and using the included alternative statistical test, likelihood ratio test, should be performed to confirm the appropriateness of these default methods.

Examining all significant results gene by gene would be impractical and examining only a subset would likely miss important biological impacts. Analysis geared towards analyzing large sets of genes was employed instead of a gene by gene or subset of genes approach.

\section{6) Gene Set Analysis}

\section{a) DAVID enriched gene set clusters and relation to muscle tissues}

The inclusion of the sarcoplasmic reticulum organelle in DAVID enriched gene set clusters depicted in Table V. indicates some of the major effects on the soleus muscle tissue are likely tissue specific. This emphasizes the importance of tissue and even cell specific analysis as effects on specialized organelles may give insights into possible countermeasures.

There are two major weakness of the DAVID approach for this analysis. First, the tools input is limited to 3000 gene IDs [32]. This meant I needed to limit my analysis to the top 3000 most significant genes and may have missed insights from the remaining significant genes. The second weakness is that DAVID only considers the significant gene themselves meaning no expression data or relative statistical significance is considered. To address these issues, another method for gene set analysis was used to more thoroughly mine the significant genes for further insights.

\section{b) Enrichment of upregulated genes in hallmark myogenesis gene set}

GSEA takes into account the gene expression differences for all genes that were not filtered out before the Wald test. This allows GSEA to detect enrichment in genes sets not only in cases of significant gene expression differences across a select number of gene set members, but in cases where smaller gene expression changes are present across a large number of gene set members.

The hallmark myogenesis gene set include genes that are involved in skeletal muscle development. Given the focus on space flight related muscle atrophy, enrichment of upregulated genes in this set was surprising. Further exploration of specific genes upregulated in this set may explain this seemingly odd result. While muscle atrophy may be a prime concern, transition from slow twitch fibers to fast twitch fibers is also a well observed effect of both space flight and the hindlimb unloading (HU) model, often taken as a ground-based analog to microgravity [46], [47]. In contrast to the enrichment of upregulated genes for myogenesis during space flight observed in this project, Cadena et al. found the hallmark myogenesis gene set to be enriched for downregulated genes in the HU model [48]. Their analysis also found the hallmark myogenesis gene set to not be significantly enriched in either upregulated or downregulated genes; however, they used an absolute fold change $\geq 2$ cutoff in their gene set enrichment analysis, while I chose to not include a fold change cutoff in favor of detecting gene set enrichment due to more modest gene expression fold changes across a large number of gene set member genes. In either case, the HU model appears to demonstrate decreased myogenesis in comparison to space flight. This suggests a 
significant difference in outcome between space flight and HU.

A possible explanation for this preservation or upregulation of myogenesis is the difference between space flight and the HU model in terms of hindlimb locomotion. In space flight, mice were observed to ambulate using all four limbs [49]. While overall ambulation time did decrease comparing space flight to ground control, the quadripedal ambulation type remained dominant, actually increasing from just under $80 \%$ at the start of the mission to nearly $100 \%$ by the end of the mission. Additionally, a space flight unique circling behavior, involving rapid running around on the enclosure walls, indicates that the quadripedal motion involved frequent high speed direction changes. This suggests frequent bursts of muscle activity that are more consistent with fast twitch fiber activity rather than slow twitch fiber activity. In turn, this circling behavior may demand myogenesis related to fast twitch development. Additional exploration of the specific genes enriched in the hallmark myogenesis pathway should be performed to find further evidence refuting or backing up this theory.

\section{c) Other hallmark gene sets enriched for upregulated genes}

The hallmark kras signaling dn gene set consists of genes downregulated by Kras. While this project focused on the microgravity factor in space flight, this enrichment may be connected to increased susceptibility to cancer due to space radiation[50].

The hallmark hypoxia gene set consists of gene related to hypoxia, lowered levels of oxygen in the body or in specific tissues. This is consistent with other studies which observed hypoxia as a health issue in space[51]-[54]. Beheshti et al. further draws a connection between increased carbon dioxide during space flight and observed hypoxia[55].

\section{d) GSEA results and downregulated hallmark gene sets}

A number of gene sets are observed as downregulated in space flight in Table VIII. This includes the hallmark reactive oxygen species pathway which is consistent with other studies that connected radiation and reactive oxygen species pathways[27], [56].

Another group of interesting downregulated gene sets was hallmark myc targets v1 and hallmark myc targets v2. This highlights a decrease in Myc targets. MYC is downregulated by Smad3 [57]. Combined with the upregulation of SMAD3 expression, as seen in Table IX, this indicates a connection between an overall decrease in Myc target expression and increased expression of SMAD3.

Another significant connection is found between SMAD3 and myostatin. Myostatin expression is increased in the space flight condition. Increases in this growth factor are associated with decreased muscle mass. SMAD3 is an upregulator of MSTN, the gene encoding myostatin [58]. A recent study by Lee et al. confirmed that both knock outs of MSTN and pharmacological inhibition of myostatin are effective countermeasures to muscle mass loss in space flight [59]. While this project was not the first to identify an overexpression of MSTN in space flight conditions, it is exciting to see this analysis confirm this specific perturbation. This is further validation that identifying differentially expression genes can form the basis for developing effective countermeasures. Towards this goal of further exploring other disrupted functions, their associated genes and ultimately potential gene expression focused countermeasures, a biological function enrichment map was generated.

\section{e) Insights from a GO biological processes enrichment map}

Fig. 17 highlights a number of manually annotated networks of GO biological processes whose gene sets are significantly upregulated or downregulated. The largest network of upregulated processes are centered around muscle and neuron related processes. The large effect on muscle related processes was to be expected based on prior literature on muscle and disuse during space flight and simulated microgravity [22], [25], [48], [59]-[61]. Other upregulated groups include biological processes for Mammary Gland / Development and Carbohydrate Metabolism. The increase in expression for genes related to carbohydrate metabolism are interesting. In a 1981 study, Abraham et al. found rats exposed to space flight conditions to have elevated levels of glycogen in their livers [62]. Since glycogen production is a component of carbohydrate metabolism, this may suggest a relation between elevated carbohydrate me- 
tabolism related genes in muscle tissue and eventual increased glycogen stores in the liver. Interestingly, Abraham et al. found that centrifuged rats in space flight had less than half the amount of glycogen found in non-centrifuged rats in space flight. This suggests perturbations to carbohydrate metabolism may be addressed through countermeasures that mitigate the experience microgravity specifically.

The groups of downregulated GO biological processes also suggest other routes for exploring space flight effects on health. First, amino acid catabolism is downregulated. This is consistent with previous observations that both protein catabolism and synthesis were decreased in humans [16].

Gene responsible for cytoskeleton structures are also highly downregulated. Previous literature has observed cytoskeleton structural changes in human cells [63]. Similar to the observations for amino acid catabolism, this suggests the observed changes in cell cytoskeleton is related in part to changes related gene expression. Combined with potential mitochondrial disruptions (evidenced by the hallmark reactive oxygen species downregulated gene enrichment), this is suggests a connection between cytoskeletal system and mitochondria dynamics consistent with the literature [64]. Additionally, perturbed mitochondrial bioenergentics is known to play a role in impaired muscle function [65].

\section{f) Heat shock protein expression downregulation and connection to muscle atrophy}

The final two groups, ribosomes and endoplasmic reticulum/protein folding, seem under explored in relation to space flight after searching PubMed. Additionally, the heat shock proteins (HSP) on Table IX. are both downregulated and associated with protein folding according to their UniProt entries [66]. Ishihara et al. also found heat shock proteins to be downregulated in mice soleus muscle in both space flight and in simulated microgravity models [67]. In contrast, human cell, plant, Drosophila and Daphnia magna experiments in space flight found such heat shock proteins to be upregulated [68]-[71]. Cazzania et al. monitored two heat shock proteins in human endothelial cells, HSP70 and HSP27, and found initially upregulation of HSP70 at four days space flight followed by return to normal levels by day 10 [72]. They also observed an upregulation in HSP27 by day 10. Cubano and Lewis also observed an increase in HSP70 and HSP27 for human lymphocytes during a 24 hour space flight [73]. This suggests that HSP downregulation in space flight is specific to non-insect animals, as the opposite effect was observed in cells, plants, and insects.

Ishihara et al. conjectured that neural stimulation was a key component of appropriate HSP induction and loss of such stimulation in real microgravity and simulated microgravity were key reasons for a drop in HSP levels. This is further supported by a studies that observe an a loss of HSP induction due to heat stress in denervated tissue including muscles [74], [75].

This suggests a potential countermeasure. If the normal expression of HSPs plays a key role in maintaining muscle mass and neural stimulation induces normal HSPs expression, then reducing loss of neural stimulation in muscles and thus mitigating downregulation of HSPs may impart a protective effect against muscle atrophy.

\section{g) Possible influence of circadian rhythm disruption and separation from normal geomagnetic field on skeletal muscle}

The role of maintaining circadian rhythm in skeletal muscle development is integral [76]. In a knockout study disrupting circadian rhythm regulation genes, soleus skeletal muscle experienced slow to fast twitch fiber transition [77]. A similar knockout study found skeletal muscles to undergo a $40 \%$ reduction of mitochondrial volume [78]. The remaining mitochondria exhibited myopathic characteristics. These effects are consistent with major effects of space flight on skeletal muscle. Disruptions to the same genes explored in these knockout studies was characterized in space flight [79]. As such, the influence of circadian rhythm disruption is likely a significant component of space flight induced skeletal muscle changes.

While the ISS is well within the influence of the geomagnetic field, the strength and orientation of the field change as the station orbits the Earth every 90 minutes [80]. In skeletal muscle cells under the influence of a hypomagnetic field on Earth, mitochondrial activity was significantly decreased [81]. On 
the other end of magnetic field exposures, studies have shown exposure to magnetic fields stronger than the Earth's increase skeletal muscle cell growth and differentiation [82], [83]. While in vivo studies exploring the effect of magnetic fields on skeletal muscles were not found at this time, these in vitro results suggests the perturbed magnetic fields may influence skeletal muscle changes during space flight in tandem with other effects.

\section{CONCLUSION}

This project achieved a Nextflow implementation of the RCP that nearly matches the posted GeneLab results for GLDS-104. The deviations are small enough that they are likely due to factors including operating system and tool version differences rather than mistakes in the Nextflow implementation of the RCP. The major benefits of the Nextflow based implementation are that it can be configured and scaled appropriately for different high performance computing environments, monitored in real-time, and can be version controlled and shared across users. The management of pipeline software using Nextflow integration with Anaconda will greatly simplify the software installation and setup phase required for running the RCP on a new machine or cluster. Additionally, running the RCP on GLDS-104 provides a useful benchmark for similar datasets. The entire analysis of $\sim 900$ million 100 base pair pair ended reads can be performed on the COS-HPC in a single day which should enable larger scale and meta-study type analysis. This highlights the importance of connecting high performance computing to genomics and multiomics analyses through pipeline software like Nextflow.

The results from analyzing GLDS-104 were examined using well validated tools. This included a DESeq2 centered differential gene analysis and gene set centered analysis through DAVID, GSEA, and Cytoscape. In particular, the results from GLDS-104 confirm previous studies that found expression changes affecting specific genes, gene sets, and related biological processes. Perturbed biological processes can be further studied, using both significant gene and significant gene set information determine in this project as starting points. This demonstrates the utility of the RCP and Nextflow implemention in developing new insights for space biology.

A theory explaining the upregulation of myogenesis related genes, notabely absent in the HU model was presented. In this theory, upregulation of myogenesis was connected to space flight unique circling behavior inducing fast twitch fiber development. While both space flight and the HU model induce slow to fast twitch fiber transitions, space flight unique ambulation may induce related adaptions to specific to space flight.

The perturbation of heat shock proteins was found to be consistent with previous studies using both space flight and simulated space flight. A possible countermeasure was discussed although additional research into the connection between HSPs and neural stimulation will need to be performed.

Finally, this project reviewed literature about circadian rhythm disruptions and magnetic field changes that likely play a confounding and/or complementary role in microgravity mediated skeletal muscle changes. 


\section{REFERENCES}

[1] R. M. Smith, M. Gates, A. Cassady, and J. Krezel, "An overview of NASA's exploration mission 2 (EM-2)," in IEEE Aerospace Conference Proceedings, Jun. 2018, vol. 2018-March, pp. 1-11, doi: 10.1109/AERO.2018.8396585.

[2] M. Weinzierl, "Space, the final economic frontier," Journal of Economic Perspectives, vol. 32, no. 2. American Economic Association, pp. 173-192, Mar. 01, 2018, doi: 10.1257/jep.32.2.173.

[3] B. J. Guzek, J. F. Horton, and C. R. Joyner, "Analyzing mission opportunities for earth to mars roundtrip missions," Adv. Astronaut. Sci., vol. 169, no. August, pp. 459-486, 2019.

[4] W. R. Carpentier et al., "Biomedical findings from NASA's project mercury: A case series," npj Microgravity, vol. 4, no. 1, p. 6, Dec. 2018, doi: 10.1038/s41526-018-0040-5.

[5] R. Jandial, R. Hoshide, J. D. Waters, and C. L. Limoli, "Space-brain: The negative effects of space exposure on the central nervous system," Surgical Neurology International, vol. 9, no. 1. Medknow Publications, Jan. 01, 2018, doi: 10.4103/sni.sni_250_17.

[6] M. M. Acharya et al., "New concerns for neurocognitive function during deep space exposures to chronic, low dose-rate, neutron radiation," eNeuro, vol. 6, no. 4, Jul. 2019, doi: 10.1523/ ENEURO.0094-19.2019.

[7] Y. Sambandam et al., "Microarray profile of gene expression during osteoclast differentiation in modelled microgravity," J. Cell. Biochem., vol. 111, no. 5, pp. 1179-1187, Dec. 2010, doi: 10.1002/ jcb.22840.

[8] V. Mann, A. Sundaresan, S. Mehta, B. Crucian, M. Doursout, and S. Devakottai, "Effects of microgravity and other space stressors in immunosuppression and viral reactivation with potential nervous system involvement," Neurol. India, vol. 67, no. 8, pp. S198-S203, May 2019, doi: 10.4103/00283886.259125 .

[9] "Rodent Research for Human Health, in Space and on Earth | NASA." https:/www.nasa.gov/ames/ rodent-research (accessed Aug. 18, 2020).

[10] W. R. Frontera and J. Ochala, "Skeletal Muscle: A Brief Review of Structure and Function," Behavior Genetics, vol. 45, no. 2. Springer New York LLC, pp. 183-195, Mar. 04, 2015, doi: 10.1007/ s00223-014-9915-y.

[11] L. M. Biga et al., 10.2 Skeletal Muscle. OpenStax/Oregon State University.

[12] G. C. Demontis, M. M. Germani, E. G. Caiani, I. Barravecchia, C. Passino, and D. Angeloni, "Human pathophysiological adaptations to the space environment," Frontiers in Physiology, vol. 8, no. AUG. Frontiers Media S.A., Aug. 02, 2017, doi: 10.3389/fphys.2017.00547.

[13] P. M. Droppert, "A review of muscle atrophy in microgravity and during prolonged bed rest.," Journal of the British Interplanetary Society, vol. 46, no. 3. pp. 83-86, 1993.

[14] K. Tanaka, N. Nishimura, and Y. Kawai, "Adaptation to microgravity, deconditioning, and countermeasures," Journal of Physiological Sciences, vol. 67, no. 2. Springer Tokyo, pp. 271-281, Mar. 01, 2017, doi: 10.1007/s12576-016-0514-8.

[15] Y. V. Gritsyna et al., "Changes in gene expression and content of Hsp70 and Hsp90 in striated mus- 
cles of mice after 30-day space flight on the biosatellite Bion-M1," Dokl. Biochem. Biophys., vol. 463, no. 1, pp. 199-202, Jul. 2015, doi: 10.1134/S1607672915040018.

[16] A. A. Ferrando, D. Paddon-Jones, and R. R. Wolfe, "Alterations in protein metabolism during space flight and inactivity," Nutrition, vol. 18, no. 10, pp. 837-841, 2002, doi: 10.1016/S08999007(02)00930-9.

[17] T. M. Mirzoev, "Skeletal muscle recovery from disuse atrophy: Protein turnover signaling and strategies for accelerating muscle regrowth," International Journal of Molecular Sciences, vol. 21, no. 21. MDPI AG, pp. 1-34, Nov. 01, 2020, doi: 10.3390/ijms21217940.

[18] R. H. Fitts et al., "Prolonged space flight-induced alterations in the structure and function of human skeletal muscle fibres," J. Physiol., vol. 588, no. 18, pp. 3567-3592, Sep. 2010, doi: 10.1113/jphysiol.2010.188508.

[19] G. Tascher et al., "Proteome-wide Adaptations of Mouse Skeletal Muscles during a Full Month in Space," J. Proteome Res., vol. 16, no. 7, pp. 2623-2638, Jul. 2017, doi: 10.1021/ acs.jproteome.7b00201.

[20] T. Kadoguchi et al., "Angiotensin II can directly induce mitochondrial dysfunction, decrease oxidative fibre number and induce atrophy in mouse hindlimb skeletal muscle," Exp. Physiol., vol. 100, no. 3, pp. 312-322, Mar. 2015, doi: 10.1113/expphysiol.2014.084095.

[21] S. Sukhanov et al., "Angiotensin II, oxidative stress and skeletal muscle wasting," in American Journal of the Medical Sciences, 2011, vol. 342, no. 2, pp. 143-147, doi: 10.1097/ MAJ.0b013e318222e620.

[22] N. T. Theilen, G. H. Kunkel, and S. C. Tyagi, "The Role of Exercise and TFAM in Preventing Skeletal Muscle Atrophy," Journal of Cellular Physiology, vol. 232, no. 9. Wiley-Liss Inc., pp. 23482358, Sep. 01, 2017, doi: 10.1002/jcp.25737.

[23] K. A. S. Silva et al., "Angiotensin II suppresses autophagy and disrupts ultrastructural morphology and function of mitochondria in mouse skeletal muscle," J. Appl. Physiol., vol. 126, no. 6, pp. 15501562, 2019, doi: 10.1152/japplphysiol.00898.2018.

[24] S. R. Zwart, R. D. Launius, G. K. Coen, J. L. L. Morgan, J. B. Charles, and S. M. Smith, "Body mass changes during long-duration spaceflight," Aviat. Sp. Environ. Med., vol. 85, no. 9, pp. 897-904, 2014, doi: 10.3357/ASEM.3979.2014.

[25] D. Sandonà et al., "Adaptation of mouse skeletal muscle to long-term microgravity in the MDS mission," PLoS One, vol. 7, no. 3, p. e33232, Mar. 2012, doi: 10.1371/journal.pone.0033232.

[26] “GeneLab Project Overview | NASA GeneLab.” https://genelab.nasa.gov/overview (accessed Nov. $30,2020)$.

[27] E. G. Overbey et al., "NASA GeneLab RNA-Seq Consensus Pipeline: Standardized Processing of Short-Read RNA-2 Seq Data 3," bioRxiv, p. 2020.11.06.371724, Nov. 2020, doi: 10.1101/2020.11.06.371724.

[28] J. Köster and S. Rahmann, "Snakemake-a scalable bioinformatics workflow engine," Bioinformatics, vol. 28, no. 19, pp. 2520-2522, 2012, doi: 10.1093/bioinformatics/bts480.

[29] P. DI Tommaso, M. Chatzou, E. W. Floden, P. P. Barja, E. Palumbo, and C. Notredame, "Nextflow enables reproducible computational workflows," Nat. Biotechnol., vol. 35, no. 4, pp. 316-319, 2017, doi: 10.1038/nbt.3820. 
[30] G. J. G. R., "Rodent Research-1 (RR1) NASA Validation Flight: Mouse soleus muscle transcriptomic and epigenomic data.” NASA GeneLab, 2017, doi: 10.26030/em9r-w619.

[31] M. I. Love, W. Huber, and S. Anders, "Moderated estimation of fold change and dispersion for RNAseq data with DESeq2,” Genome Biol., vol. 15, no. 12, p. 550, Dec. 2014, doi: 10.1186/s13059-0140550-8.

[32] D. W. Huang, B. T. Sherman, and R. A. Lempicki, "Systematic and integrative analysis of large gene lists using DAVID bioinformatics resources," Nat. Protoc., vol. 4, no. 1, pp. 44-57, 2009, doi: 10.1038/nprot.2008.211.

[33] A. Subramanian et al., "Gene set enrichment analysis: A knowledge-based approach for interpreting genome-wide expression profiles," Proc. Natl. Acad. Sci. U. S. A., vol. 102, no. 43, pp. 15545-15550, Oct. 2005, doi: 10.1073/pnas.0506580102.

[34] M. Martin, "Cutadapt removes adapter sequences from high-throughput sequencing reads," EMBnet.journal, vol. 17, no. 1, p. 10, May 2011, doi: 10.14806/ej.17.1.200.

[35] P. Ewels, M. Magnusson, S. Lundin, and M. Käller, "MultiQC: summarize analysis results for multiple tools and samples in a single report," Bioinformatics, vol. 32, no. 19, pp. 3047-3048, Oct. 2016, doi: 10.1093/bioinformatics/btw354.

[36] A. Dobin et al., "STAR: ultrafast universal RNA-seq aligner," Bioinformatics, vol. 29, no. 1, pp. 1521, Jan. 2013, doi: 10.1093/bioinformatics/bts635.

[37] B. Li and C. N. Dewey, "RSEM: Accurate transcript quantification from RNA-Seq data with or without a reference genome," BMC Bioinformatics, vol. 12, no. 1, p. 323, Aug. 2011, doi: 10.1186/14712105-12-323.

[38] A. D. Yates et al., "Ensembl 2020," Nucleic Acids Res., vol. 48, no. D1, pp. D682-D688, Jan. 2020, doi: 10.1093/nar/gkz966.

[39] S. Anders and W. Huber, "Differential expression analysis for sequence count data," Genome Biol., vol. 11, no. 10, p. R106, Oct. 2010, doi: 10.1186/gb-2010-11-10-r106.

[40] S. Carbon et al., "The Gene Ontology Resource: 20 years and still GOing strong," Nucleic Acids Res., vol. 47, no. D1, pp. D330-D338, Jan. 2019, doi: 10.1093/nar/gky1055.

[41] P. Shannon et al., "Cytoscape: A software Environment for integrated models of biomolecular interaction networks," Genome Res., vol. 13, no. 11, pp. 2498-2504, Nov. 2003, doi: 10.1101/gr.1239303.

[42] D. Merico, R. Isserlin, O. Stueker, A. Emili, and G. D. Bader, "Enrichment Map: A Network-Based Method for Gene-Set Enrichment Visualization and Interpretation," PLoS One, vol. 5, no. 11, p. e13984, Nov. 2010, doi: 10.1371/journal.pone.0013984.

[43] C. Franzini-Armstrong and A. G. Engel, "Skeletal muscle: Architecture of membrane systems," in Muscle, vol. 2, Elsevier Inc., 2012, pp. 763-774.

[44] C. R. Williams, A. Baccarella, J. Z. Parrish, and C. C. Kim, "Trimming of sequence reads alters RNA-Seq gene expression estimates,” BMC Bioinformatics, 2016, doi: 10.1186/s12859-016-0956-2.

[45] L. Wang, S. Wang, and W. Li, "RSeQC: Quality control of RNA-seq experiments," Bioinformatics, vol. 28, no. 16, pp. 2184-2185, Aug. 2012, doi: 10.1093/bioinformatics/bts356.

[46] S. Pierno et al., "Potential benefits of taurine in the prevention of skeletal muscle impairment induced by disuse in the hindlimb-unloaded rat," Amino Acids, vol. 43, no. 1, pp. 431-445, Jul. 2012, doi: 
[47] J. F. Desaphy, S. Pierno, C. Léoty, A. L. George, A. De Luca, and D. C. Camerino, "Skeletal muscle disuse induces fibre type-dependent enhancement of $\mathrm{Na}+$ channel expression," Brain, vol. 124, no. 6, pp. 1100-1113, Jun. 2001, doi: 10.1093/brain/124.6.1100.

[48] S. M. Cadena et al., "Skeletal muscle in MuRF1 null mice is not spared in low-gravity conditions, indicating atrophy proceeds by unique mechanisms in space," Sci. Rep., vol. 9, no. 1, p. 9397, 2019, doi: 10.1038/s41598-019-45821-9.

[49] A. E. Ronca et al., "Behavior of mice aboard the International Space Station," Sci. Rep., vol. 9, no. 1, Dec. 2019, doi: 10.1038/s41598-019-40789-y.

[50] M. H. Barcellos-Hoff et al., "Concepts and challenges in cancer risk prediction for the space radiation environment," Life Sciences in Space Research, vol. 6. Elsevier Ltd, pp. 92-103, Jul. 01, 2015, doi: 10.1016/j.1ssr.2015.07.006.

[51] E. L. Moyer et al., "Evaluation of rodent spaceflight in the NASA animal enclosure module for an extended operational period (Up to 35 days)," npj Microgravity, vol. 2, no. 1, p. 16002, Jan. 2016, doi: 10.1038/npjmgrav.2016.2.

[52] A. Beheshti et al., "Exploring the effects of spaceflight on mouse physiology using the open access NASA genelab platform,” J. Vis. Exp., vol. 2019, no. 143, p. 58447, Jan. 2019, doi: 10.3791/58447.

[53] C. G. T. Tahimic and R. K. Globus, "Redox signaling and its impact on skeletal and vascular responses to spaceflight," International Journal of Molecular Sciences, vol. 18, no. 10. MDPI AG, Oct. 16, 2017, doi: 10.3390/ijms18102153.

[54] R. L. Hughson, A. Helm, and M. Durante, "Heart in space: Effect of the extraterrestrial environment on the cardiovascular system," Nature Reviews Cardiology, vol. 15, no. 3. Nature Publishing Group, pp. 167-180, Mar. 01, 2018, doi: 10.1038/nrcardio.2017.157.

[55] A. Beheshti, E. Cekanaviciute, D. J. Smith, and S. V. Costes, "Global transcriptomic analysis suggests carbon dioxide as an environmental stressor in spaceflight: A systems biology GeneLab case study," Sci. Rep., vol. 8, no. 1, Dec. 2018, doi: 10.1038/s41598-018-22613-1.

[56] A. Beheshti, J. T. McDonald, J. Miller, P. Grabham, and S. V. Costes, "Genelab database analyses suggest long-term impact of space radiation on the cardiovascular system by the activation of FYN through reactive oxygen species," Int. J. Mol. Sci., vol. 20, no. 3, Feb. 2019, doi: 10.3390/ ijms20030661.

[57] C. R. Chen, Y. Kang, P. M. Siegel, and J. Massagué, "E2F4/5 and p107 as Smad cofactors linking the TGF $\beta$ receptor to c-myc repression," Cell, vol. 110, no. 1, pp. 19-32, Jul. 2002, doi: 10.1016/S00928674(02)00801-2.

[58] D. L. Allen and T. G. Unterman, "Regulation of myostatin expression and myoblast differentiation by FoxO and SMAD transcription factors," Am. J. Physiol. - Cell Physiol., vol. 292, no. 1, Jan. 2007, doi: 10.1152/ajpcell.00542.2005.

[59] S. J. Lee et al., "Targeting myostatin/activin A protects against skeletal muscle and bone loss during spaceflight," Proc. Natl. Acad. Sci. U. S. A., vol. 117, no. 38, pp. 23942-23951, Sep. 2020, doi: 10.1073/pnas.2014716117.

[60] T. Uchida et al., "Reactive oxygen species upregulate expression of muscle atrophy-associated ubiquitin ligase Cbl-b in rat L6 skeletal muscle cells," Am. J. Physiol. - Cell Physiol., vol. 314, no. 6, pp. 
C721-C731, Jun. 2018, doi: 10.1152/ajpcell.00184.2017.

[61] C. Däpp, S. Schmutz, H. Hoppeler, and M. Flück, “Transcriptional reprogramming and ultrastructure during atrophy and recovery of mouse soleus muscle," Physiol. Genomics, vol. 20, no. 1, pp. 97-107, Apr. 2005, doi: 10.1152/physiolgenomics.00100.2004.

[62] S. Abraham, C. Y. Lin, H. P. Klein, and C. Volkmann, "The effects of space flight on some rat liver enzymes regulating carbohydrate and lipid metabolism,” Adv. Sp. Res., vol. 1, no. 14, pp. 199-217, 1981, doi: 10.1016/0273-1177(81)90263-5.

[63] T. J. Corydon et al., "Alterations of the cytoskeleton in human cells in space proved by life-cell imaging,” Sci. Rep., vol. 6, Jan. 2016, doi: 10.1038/srep20043.

[64] L. Locatelli, A. Cazzaniga, C. De Palma, S. Castiglioni, and J. A. M. Maier, "Mitophagy contributes to endothelial adaptation to simulated microgravity," FASEB J., vol. 34, no. 1, pp. 1833-1845, Jan. , doi: 10.1096/fj.201901785RRR.

[65] X. Zhang et al., "Impaired Mitochondrial Energetics Characterize Poor Early Recovery of Muscle Mass Following Hind Limb Unloading in Old Mice," Journals Gerontol. - Ser. A Biol. Sci. Med. Sci., vol. 73, no. 10, pp. 1313-1322, Sep. 2018, doi: 10.1093/gerona/gly051.

[66] A. Bateman, "UniProt: A worldwide hub of protein knowledge," Nucleic Acids Res., vol. 47, no. D1, pp. D506-D515, Jan. 2019, doi: 10.1093/nar/gky1049.

[67] A. Ishihara, H. Fujino, F. Nagatomo, I. Takeda, and Y. Ohira, "Gene expression levels of heat shock proteins in the soleus and plantaris muscles of rats after hindlimb suspension or spaceflight," J. Physiol. Sci., vol. 58, no. 6, pp. 413-417, 2008, doi: 10.2170/physiolsci.RP000808.

[68] B. Trotter et al., "The influence of simulated microgravity on the proteome of Daphnia magna," npj Microgravity, vol. 1, no. 1, p. 1, Sep. 2015, doi: 10.1038/njjmgrav.2015.16.

[69] K. Taylor et al., "Toll mediated infection response is altered by gravity and spaceflight in Drosophila," PLoS One, vol. 9, no. 1, Jan. 2014, doi: 10.1371/journal.pone.0086485.

[70] A. K. Zupanska, F. C. Denison, R. J. Ferl, and A. L. Paul, "Spaceflight engages heat shock protein and other molecular chaperone genes in tissue culture cells of Arabidopsis Thaliana," Am. J. Bot., vol. 100, no. 1, pp. 235-248, Jan. 2013, doi: 10.3732/ajb.1200343.

[71] T. G. Hammond et al., "Mechanical culture conditions effect gene expression: Gravity-induced changes on the space shuttle," Physiol. Genomics, vol. 2000, no. 3, pp. 163-173, 2000, doi: 10.1152/ physiolgenomics.2000.3.3.163.

[72] A. Cazzaniga, L. Locatelli, S. Castiglioni, and J. A. M. Maier, "The dynamic adaptation of primary human endothelial cells to simulated microgravity," FASEB J., vol. 33, no. 5, pp. 5957-5966, May 2019, doi: 10.1096/fj.201801586RR.

[73] L. A. Cubano and L. Lewis, "Effect of vibrational stress and spaceflight on regulation of heat shock proteins hsp70 and hsp27 in human lymphocytes (Jurkat)," J. Leukoc. Biol., vol. 69, no. 5, pp. 75575561, May 2001, doi: 10.1189/jlb.69.5.755.

[74] K. Yamada, S. Goto, and Y. Ushio, "Occurrence of heat shock response in deafferented neurons in the substantia nigra of rats," Neuroscience, vol. 62, no. 3, pp. 793-801, Oct. 1994, doi: 10.1016/0306-4522(94)90477-4.

[75] T. Ohira et al., "The effects of heat stress on morphological properties and intracellular signaling of denervated and intact soleus muscles in rats," Physiol. Rep., vol. 5, no. 15, Aug. 2017, doi: 10.14814/ 
phy2.13350.

[76] B. D. Harfmann, E. A. Schroder, and K. A. Esser, "Circadian rhythms, the molecular clock, and skeletal muscle," Journal of Biological Rhythms, vol. 30, no. 2. SAGE Publications Inc., pp. 84-94, Apr. 01, 2015, doi: 10.1177/0748730414561638.

[77] K. A. Dyar et al., "Muscle insulin sensitivity and glucose metabolism are controlled by the intrinsic muscle clock," Mol. Metab., vol. 3, no. 1, pp. 29-41, Feb. 2014, doi: 10.1016/j.molmet.2013.10.005.

[78] J. L. Andrews et al., "CLOCK and BMAL1 regulate MyoD and are necessary for maintenance of skeletal muscle phenotype and function," Proc. Natl. Acad. Sci. U. S. A., vol. 107, no. 44, pp. 19090 19095, Nov. 2010, doi: 10.1073/pnas.1014523107.

[79] S. I. Fujita, L. Rutter, Q. Ong, and M. Muratani, "Integrated RNA-seq analysis indicates asynchrony in clock genes between tissues under spaceflight," Life, vol. 10, no. 9, pp. 1-12, Sep. 2020, doi: 10.3390/life10090196.

[80] C. Del Seppia, L. Mezzasalma, M. Messerotti, A. Cordelli, and S. Ghione, "Simulation of the geomagnetic field experienced by the International Space Station in its revolution around the Earth: Effects on psychophysiological responses to affective picture viewing," Neurosci. Lett., vol. 400, no. 3, pp. 197-202, Jun. 2006, doi: 10.1016/j.neulet.2006.02.045.

[81] J. P. Fu, W. C. Mo, Y. Liu, and R. Q. He, "Decline of cell viability and mitochondrial activity in mouse skeletal muscle cell in a hypomagnetic field," Bioelectromagnetics, vol. 37, no. 4, pp. 212 222, 2016, doi: 10.1002/bem.21968.

[82] S. V. Surma, G. B. Belostotskaya, B. F. Shchegolev, and V. E. Stefanov, "Effect of weak static magnetic fields on the development of cultured skeletal muscle cells," Bioelectromagnetics, vol. 35, no. 8, pp. 537-546, 2014, doi: 10.1002/bem.21876.

[83] D. Coletti et al., "Static magnetic fields enhance skeletal muscle differentiation in vitro by improving myoblast alignment," Cytom. Part A, vol. 71, no. 10, pp. 846-856, 2007, doi: 10.1002/ cyto.a.20447. 